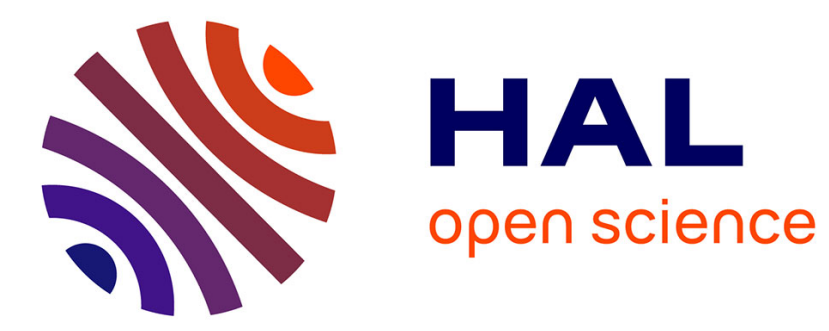

\title{
Diagnosability of Repairable Faults
}

\author{
Eric Fabre, Loïc Hélouët, Engel Lefaucheux, Hervé Marchand
}

\section{To cite this version:}

Eric Fabre, Loïc Hélouët, Engel Lefaucheux, Hervé Marchand. Diagnosability of Repairable Faults. Discrete Event Dynamic Systems, 2018, 28 (2), pp.183-213. 10.1007/s10626-017-0255-8 . hal01646911

\section{HAL Id: hal-01646911 https://hal.inria.fr/hal-01646911}

Submitted on 23 Nov 2017

HAL is a multi-disciplinary open access archive for the deposit and dissemination of scientific research documents, whether they are published or not. The documents may come from teaching and research institutions in France or abroad, or from public or private research centers.
L'archive ouverte pluridisciplinaire HAL, est destinée au dépôt et à la diffusion de documents scientifiques de niveau recherche, publiés ou non, émanant des établissements d'enseignement et de recherche français ou étrangers, des laboratoires publics ou privés. 


\title{
Diagnosability of Repairable Faults
}

\author{
Eric Fabre · Loïc Hélouët · Engel Lefaucheux · \\ Hervé Marchand
}

Received: date / Accepted: date

\begin{abstract}
The diagnosis problem for discrete event systems consists in deciding whether some fault event occurred or not in the system, given partial observations on the run of that system. Diagnosability checks whether a correct diagnosis can be issued in bounded time after a fault, for all faulty runs of that system. This problem appeared two decades ago and numerous facets of it have been explored, mostly for permanent faults. It is known for example that diagnosability of a system can be checked in polynomial time, while the construction of a diagnoser is exponential. The present paper examines the case of transient faults, that can appear and be repaired. Diagnosability in this setting means that the occurrence of a fault should always be detected in bounded time, but also before the fault is repaired. Checking this notion of diagnosability is proved to be PSPACE-complete. It is also shown that faults can be reliably counted provided the system is diagnosable for faults and for repairs.
\end{abstract}

\section{Introduction}

The diagnosis problem for discrete event systems appeared two decades ago [10]. In its standard version the problem assumes a dynamic system $A$ with runs of two types: some runs are safe (they contain no fault event), and the others are faulty. More generally, one may assume a regular property $P$ on runs of $A$. This property $P$ is absorbing, in the sense that once $P$ is satisfied by some partial run (like the fact of being faulty) it remains true in all extensions of that run. System $A$ is supposed to perform some hidden run $u$, which is partially observed by an external supervisor: only some events of the hidden run $u$ are visible, possibly through some filtering operation, and the other events of $u$ are silent. The problem then consists in deciding whether the hidden run $u$ satisfies the property $P$ of interest given the observed sequence and the model of $A$. Specifically, assuming that $u$ satisfies $P$ at some instant $t$, one would like to detect that $P$ holds in bounded time after $t$. If this is feasible for all runs satisfying $P$, the system is declared diagnosable.

INRIA Rennes, Campus de Beaulieu, 35042 Rennes cedex, France name.surnamedinria.fr 
Property $P$ can be seen as an abstraction on the behaviors of $A$, which are partially observed. Diagnosability then amounts to detecting when $P$ holds. One may further be interested in deciding in bounded time after time $t$ whether $P$ holds or not at time $t$. A dual version of the problem relates to opacity: one would like to ensure that property $P$ (a "secret") is never detectable by an external observer. Beyond its simple statement, the diagnosis problem thus has numerous implications in terms of security and of safety.

Numerous facets of the diagnosis problem have been explored since its first introduction: We refer the reader to [13] for a clear review of the different settings, with various kinds of modeling formalisms for both systems and faults. In this paper, we examine the case of non persistent properties $P$, or non persistent faults, i.e. $P$ may hold only on segments of the hidden run $u$. Diagnosing $P$ thus means being able to detect that $P$ holds in bounded time after it becomes true, and in any case before $P$ vanishes. Section 2 recalls standard results about the classical notion of diagnosability. The diagnosability for repairable faults, named T-diagnosability, is presented in Section 3. It is proved that deciding T-diagnosability is PSPACE-complete. Section 4 expresses that when both faults and repairs are T-diagnosable, one is able to count fault occurrences in the hidden run $u$. Finally, Section 5 relates these results to previous contributions on the topic, in particular to the notion of P-diagnosability introduced by [3] and which is closely related to our notion of T-diagnosability. It is proved that deciding P-diagnosability is also a PSPACE-complete problem.

In this paper, we extend [4] in several directions: In particular, we provide algorithms in NLOGSPACE allowing to check the presence of vanishing faults in the systems. We also show how to remove the vanishing faults/repairs from a system assuming that this kind of faults might be out-of interest for diagnosis purposes. Finally, we compare our notion of T-Diagnosability with the P-diagnosability introduced by [3] ans proved that checking the latter is also a PSPACE-complete problem. The proofs of Section 4 have also been added.

\section{Setting and known results}

\subsection{Diagnosis and diagnoser}

Let $\Sigma$ be a finite alphabet. A word over $\Sigma$ is a element $w \in \sigma^{*}$. We denote by $\varepsilon$ the empty word. Given a pair of words $u, v \in \sigma^{*}$, we will write $u \leq v$ when $u$ is a prefix of $v$, i.e. $v=u . u^{\prime}$. The length of a word $v \in \sigma^{*}$ is denoted $|v|$. A finite automaton over alphabet $\Sigma$ is a tuple $A=\left(S, \Sigma, T, s_{0}\right)$, where $S$ is a finite set of states, $s_{0} \in S$ is the initial state, and $T \subseteq S \times \Sigma \times S$ is a set of transitions. Transitions take the form $t=\left(s, \alpha, s^{\prime}\right)$ and we denote $s^{-}(t)=s, \sigma(t)=\alpha, s^{+}(t)=s^{\prime}$. Paths of $A$ are finite sequences of transitions $u=t_{1} \ldots t_{n}$ such that $s^{+}\left(t_{i}\right)=s^{-}\left(t_{i+1}\right)$, and runs of $A$ are paths rooted at $s_{0}: s^{-}\left(t_{1}\right)=s_{0}$. We denote $s^{-}(u)=s^{-}\left(t_{1}\right), s^{+}(u)=s^{+}\left(t_{n}\right)$, and $\sigma(u)=\sigma\left(t_{1}\right) \ldots \sigma\left(t_{n}\right)$ the sequence of labels associated to a path $u$. A path $u=t_{1} \ldots t_{n}$ is reachable iff there exists a run $v$ of $A$ such that $s^{+}(v)=s^{-}(u)$. A path $u$ is a cycle iff $s^{+}(u)=s^{-}(u)$. The language of $A$ is the set of label sequences produced by runs of 
$A: L(A)=\{\sigma(u), u$ run of $A\}$. An automaton is deterministic iff $\forall s, \alpha,\left(s, \alpha, s^{\prime}\right) \in$ $T \wedge\left(s, \alpha, s^{\prime \prime}\right) \in T \Rightarrow s^{\prime}=s^{\prime \prime}$.

Our starting point for the diagnosis problem, and without loss of generality, is a deterministic automaton $A$. Let us partition states of $A$ into two subsets $S=S_{N} \uplus S_{F}$, and let us name $S_{N}$ normal (or safe) states and $S_{F}$ faulty states, to help intuition. The faulty language of $A$ is derived from faulty runs, i.e. runs that terminate in a faulty state: $L_{F}(A)=\left\{\sigma(u), u\right.$ run of $\left.A, s^{+}(u) \in S_{F}\right\}$. The normal (safe) language of $A$ is denoted $L_{N}(A)$, and is defined in similar way as $L_{N}(A)=\left\{\sigma(u), u\right.$ run of $A, s^{+}(u) \in$ $\left.S_{N}\right\}$. Obviously, we have $L_{F}(A) \subseteq L(A)$ and $L_{F}(A) \subseteq L(A)$. As $A$ is deterministic, $\sigma$ establishes a one to one correspondence between runs of $A$ and words of its language $L(A)$, so $L_{N}(A) \cap L_{F}(A)=\emptyset$, or $L_{N}(A) \uplus L_{F}(A)=L(A)$. In this section, we assume that faults are permanent in $A$. Namely, there is no reachable path $u$ in $A$ such that $s^{-}(u) \in S_{F}$ and $s^{+}(u) \in S_{N}$. Equivalently, the faulty language of $A$ is saturated in $L(A): L_{F}(A) \Sigma^{*} \cap L(A)=L_{F}(A)$.

The diagnosis problem assumes partially observed systems, so we partition the label set $\Sigma$ in two disjoint sets of observable and unobservable labels: $\Sigma=\Sigma_{o} \uplus \Sigma_{u}$. The projection on observable labels $\Pi: \Sigma^{*} \rightarrow \Sigma_{o}^{*}$ is defined as the monoid morphism generated by $\Pi(\alpha)=\alpha$ whenever $\alpha \in \Sigma_{o}$ and $\Pi(\alpha)=\varepsilon$ otherwise. Given $v \in \Sigma^{*}$, we denote by $|v|_{o}$ the number of observable events in $w$ i.e. $|w|_{o}=|\Pi(w)|$.

The observable (or visible) language of $A$ is defined as $L_{o}(A)=\Pi(L(A))$. For technical reasons commented later, we define the inverse projection $\Pi^{-1}$ as follows :

$$
\forall w \in \Sigma_{o}^{*}, \Pi^{-1}(w)=\{v \in L(A): \Pi(v)=w\} \cap \Sigma^{*} \Sigma_{o}
$$

i.e. we restrict the standard inverse projection to words of $L(A)$ that end with an observable letter ${ }^{1}$.

From a run $u$ performed by $A$, or equivalently from the word $v=\sigma(u)$, one only observes the visible actions i.e. the word $w=\Pi(v)=\sigma_{o}(u) \in L_{o}(A)$, where $\sigma_{o}=$ $\Pi \circ \sigma$. Note that a given observed word $w \in \Sigma_{o}^{*}$ might be the observation (i.e., the projection) of any word in $\Pi^{-1}(w)$. The diagnosis consists in deciding whether a fault has occurred in system $A$ given this observed sequence $w$. A diagnoser for $A$ can be seen as a function $\Delta: L_{o}(A) \rightarrow\{N, F, U\}$ where

$$
\Delta(w)=\left\{\begin{array}{l}
N \text { iff } \Pi^{-1}(w) \subseteq L_{N}(A) \\
F \text { iff } \Pi^{-1}(w) \subseteq L_{F}(A) \\
U \text { otherwise }
\end{array}\right.
$$

Letters $N, F, U$ stand for "normal", "faulty", and "uncertain", (or "ambiguous") as it clearly appears above.

A diagnoser can be derived from an observer (or state estimator) of $A$. This observer is built in two steps. The first step is the $\Sigma_{o}$-closure of $A$. The $\Sigma_{o}$-closure (to the left) of $A$ is defined as $B=\operatorname{Red}_{\Sigma_{o}}(A)=\left(S, \Sigma_{o}, T^{\prime}, s_{0}\right)$ where $\left(s, \alpha, s^{\prime}\right) \in T^{\prime}$ iff there exists a path $u=t_{1} \ldots t_{n}$ in $A$ such that $\sigma_{o}\left(t_{1} \ldots t_{n-1}\right)=\varepsilon, \sigma_{o}\left(t_{n}\right)=\alpha, s^{-}(u)=s$ and $s^{+}(u)=s^{\prime}$. Intuitively, there is a transition from $s$ to $s^{\prime}$ in $T^{\prime}$ iff there exists a path

\footnotetext{
1 Alternatively, we can define $L(A)$ as words that terminate with a letter of $\Sigma_{o}$, or equivalently by assuming faulty states in $A$ that can only be reached by visible transitions, which does not reduce the generality of the setting.
} 
from $s$ to $s^{\prime}$ with a single observable action labeling the last transition of the path. The $\Sigma_{o}$-closure of $A$ is an $\varepsilon$-reduction (to the left) assuming all labels of $\Sigma_{u}$ are first replaced by $\varepsilon$ in $A$. The second step needed to build a state estimator for $A$ is the determinization of the resulting $B$, performed by standard subset construction. Let $D=\operatorname{Det}(B)=\left(Q, \Sigma_{o}, T^{\prime \prime}, q_{0}\right)$ where $Q=2^{S}, q_{0}=\left\{s_{0}\right\}$ and $t=\left(q, \alpha, q^{\prime}\right) \in T^{\prime \prime}$ iff $q^{\prime}=\left\{s^{\prime} \in S: \exists s \in q,\left(s, \alpha, s^{\prime}\right) \in T^{\prime}\right\}$. Of course, both $B$ and $D$ can be trimmed to their reachable part.

Observe that $L(D)=L(B)=L_{o}(A) . D$ is a state estimator of $A$ in the following sense : let $w \in L_{o}(A)$, as $D$ is deterministic, there exists a unique path $r$ in $D$ such that $\sigma_{o}(r)=w$. The final state $q=s^{+}(r) \in Q$ of path $r$ in $D$ satisfies $q=s^{+}\left(\sigma_{o}^{-1}(w)\right) \in 2^{S}$ in $A$, i.e. it contains all states of $A$ that are reachable by runs that produce the observed sequence $w$ and that stop immediately after the last observable transition. This last condition explains the specific definition of $\Pi^{-1}$ and the choice of the $\Sigma_{o}$-closure of A to the left. Let us call $q \in Q=2^{S}$ a normal subset iff $q \subseteq S_{N}$, a faulty subset iff $q \subseteq S_{F}$, and an uncertain (or ambiguous) subset otherwise. $D$ yields a diagnoser for $A$ as follows : $\Delta(w)$ is the type of $q=s^{+}\left(\sigma_{o}^{-1}(w)\right)$ in $D$. By extension, $D$ is often called the diagnoser of $A: D=\operatorname{Diag}(A)=\operatorname{Det}\left(\operatorname{Red}_{\Sigma_{o}}(A)\right)$.

Due to determinization, $D$ can be exponentially larger than $A$ and should not be used for online diagnosis. One should use instead a recursive state estimation driven by the observed sequence $w$, which has linear complexity in the size of $w$ and $A$. $D$ can thus be considered as a precompiled version of the diagnosis for all possible observed sequences.

\subsection{Remarks and extensions}

Let $A_{1}, A_{2}$ be two automata, with $A_{i}=\left(S_{i}, \Sigma_{i}, T_{i}, s_{0, i}\right)$, their synchronous product (or simply product for short) is the automaton $A_{1} \times A_{2}=\left(S_{1} \times S_{2}, \Sigma_{1} \cup \Sigma_{2}, T_{1} \otimes\right.$ $\left.T_{2},\left(s_{1,0}, s_{2,0}\right)\right)$ where transitions in $T_{1} \otimes T_{2}$ are triples $\left(\left(s_{1}, s_{2}\right), \alpha,\left(s_{1}^{\prime}, s_{2}^{\prime}\right)\right)$ such that

$$
\begin{gathered}
\left(s_{1}, \alpha, s_{1}^{\prime}\right) \in T_{1} \wedge\left(s_{2}, \alpha, s_{2}^{\prime}\right) \in T_{2} \text { for } \alpha \in \Sigma_{1} \cap \Sigma_{2} \\
\left(s_{1}, \alpha, s_{1}^{\prime}\right) \in T_{1} \wedge s_{2}=s_{2}^{\prime} \in S_{2} \text { for } \alpha \in \Sigma_{1} \backslash \Sigma_{2} \\
s_{1}=s_{1}^{\prime} \in S_{1} \wedge\left(s_{2}, \alpha, s_{2}^{\prime}\right) \in T_{2} \text { for } \alpha \in \Sigma_{2} \backslash \Sigma_{1}
\end{gathered}
$$

Faulty runs in $A$ are often not identified by a partition on states, but rather by the firing of some transition carrying a "fault" label $f \in \Sigma_{u}$. This can be recast in the previous setting as follows. Consider the deterministic and complete memory automaton $M=(\{N, F\}, \Sigma, T, N)$ of Figure 1 where $(N, f, F)$ is the unique transition of $T$ producing a state change. The product $A \times M$ does not change the language of $A$, but performs a state augmentation that keeps track of the firing of a faulty transition in $A$. The label $N$ or $F$ now attached to states of $A \times M$ defines a partition of the state set that characterizes faulty runs. This technique was generalized in [5] to detect/diagnose runs satisfying some regular pattern of labels, rather than the simple firing of a transition labeled by $f$.

When faults are non permanent in $A$, that is when there exist transitions from $S_{F}$ to $S_{N}$, one may nevertheless be interested in detecting that some transient fault 


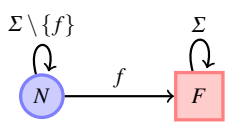

Fig. 1 A fault detection automaton.

has occurred, i.e. a fault followed by a repair. This can again be handled as a standard diagnosis problem: one can transform of $A$ into another automaton $A^{\prime}$, that adds memory to states of $A$ to propagate the fact that a fault occurred sometime in the past, and then build a diagnoser for $A^{\prime}$. With the assumption that $A$ is deterministic, this amounts to saturating the fault language of $A: L_{F}\left(A^{\prime}\right)=L_{F}(A) \Sigma^{*} \cap L(A)$. A diagnoser for $A^{\prime}$ detects faulty runs of $A^{\prime}$, i.e. it also detects runs of $A$ that contain a fault. This idea is a variant of the pattern recognition of [5]. It was used in [7] to track the occurrence of $k$ transient faults. It is also present in [3] under the names of O-diagnosis (detection of the occurrence of a fault) and I-diagnosis (detection of the occurrence of a repair). All these notions are thus variants of the classical diagnosis approach, even if they are recast in the context of transient failures. In [3], the authors propose a "memory automaton" that can be composed with a specification to remember occurrences of faults and repairs. However, even if fault repair is considered, their automaton propagates the information that a fault occurred. Within this setting, past occurrences of faults can be diagnosed, but without guarantee that faults are detected while the system is faulty. In the next section, we consider a different setting, where diagnosis is considered as accurate if it detects a fault before it is repaired.

\subsection{Diagnosability}

Let us recall the notion of diagnosability for permanent faults, i.e. when $A$ has no transition from $S_{F}$ to $S_{N}$. For simplicity, we assume that $A$ is $\Sigma_{o}$-live: an observable transition is reachable from any state of $A$. Intuitively, $A$ is diagnosable iff, whenever it reaches $S_{F}$, this is detected/diagnosed after a finite number of extra observations. Formally, $A$ is diagnosable iff

$$
\begin{gathered}
\forall v_{1} \in L_{F}(A), \exists n \in \mathbb{N}, \forall v_{1} v_{2} \in L(A), \\
{\left[\left|v_{2}\right|_{o} \geq n \Rightarrow \Pi^{-1} \circ \Pi\left(v_{1} v_{2}\right) \subseteq L_{F}(A)\right]}
\end{gathered}
$$

where $\left|v_{2}\right|_{o}$ is the length of $\Pi\left(v_{2}\right)$. This expression slightly differs from more frequent ones (for ex. [10]), that count the number of transitions in $v$ rather than the number of observable transitions, (i.e. require $\left|v_{2}\right| \geq n$ instead of $\left|v_{2}\right|_{o} \geq n$ ) but remains equivalent in essence. First, Definition (3) counts only visible transitions in $\left|v_{2}\right|_{o}$, instead of counting all transitions. It makes more sense to have an observable criterion to decide when to collect the diagnosis. And when $A$ has no unobservable cycle, which is generally assumed when one uses $|v|$ instead of $|v|_{o}$, this rephrasing is harmless. Secondly, one generally assumes a uniform value of $n$ covering all faulty words $v_{1}$. Again, taking account the finiteness of $A$, this uniform bound comes for free once (3) holds. 


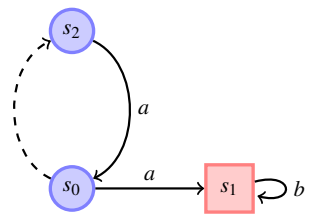

Fig. 2 Normal/faulty states are represented as circle/square boxes. The dashed line represents an unobservable transition. This automaton is diagnosable: after it reaches the faulty state, it can only produce a $b$ which characterizes the occurrence of the fault. Nevertheless, driven by sequence $a^{n}$, the diagnoser outputs $U^{n}$. So uncertainty can be arbitrarily long.

Def. (3) states that a system is diagnosable iff, when uncertainty appears after a faulty run, it does not hold forever. Observe that the diagnosis may nevertheless remain uncertain for an arbitrarily long time, even for a diagnosable system, as long as no fault occurs (see Fig. 2). Conversely, $A$ is not diagnosable iff after some faulty run uncertainty may last for an arbitrary long time :

$$
\begin{gathered}
\exists v_{1} \in L_{F}(A), \forall n \in \mathbb{N}, \exists v_{1} v_{2} \in L(A), \\
{\left[\left|v_{2}\right|_{o} \geq n \wedge \Pi^{-1} \circ \Pi\left(v_{1} v_{2}\right) \cap L_{N}(A) \neq \emptyset\right]}
\end{gathered}
$$

The last term in (4) can be rephrased as $\Delta\left(\Pi\left(v_{1} v_{2}\right)\right)=U$ or equivalently $\exists v^{\prime} \in L_{N}(A)$, $\Pi\left(v_{1} v_{2}\right)=\Pi\left(v^{\prime}\right)$. This new formulation expresses that one can find an arbitrary long extension $v_{2}$ of some faulty word $v_{1}$ which is observationally equivalent (or equivalent for short) to a safe word $v^{\prime}$ of $A$, denoted by $v_{1} v_{2} \sim_{o} v^{\prime}$. As faults are permanent, any prefix of the safe word $v^{\prime}$ is also safe. Def. (4) thus opens the way to a polynomial test for (non-)diagnosability: one can build a twin-machine that recognizes pairs of runs made of a faulty one $v_{1} v_{2}$ and an equivalent (w.r.t observation) safe one $v^{\prime}$, and thus check how long uncertainty can last.

Consider $B=\operatorname{Red}_{\Sigma_{o}}(A)$, the twin machine of $A$ is obtained as $C=B \times B$. A run in $C$ represents a pair of runs of $B$ that are observationally equivalent. Hence, for every run of $C$, there exists a pair $\left(v, v^{\prime}\right)$ of observationally equivalent words of $L(A)$. The states of $C$ is a subset of $S \times S$. So, using the same principle as in $D$, the diagnoser of $A$, a state $\left(s, s^{\prime}\right)$ of $C$ can be called normal/safe if $s$ and $s^{\prime}$ belong to $S_{N}$, faulty if $s$ and $s^{\prime}$ belong to $S_{F}$ and uncertain/ambiguous otherwise. An ambiguous cycle in $C$ is a reachable cycle that only goes through ambiguous (pairs of) states.

Proposition $1 A$ is diagnosable iff its twin machine $C$ has no ambiguous cycle.

This result was proved in [6]. The only if part is obvious as the presence of an ambiguous cycle allows one to build an arbitrarily long suffix $v_{2}$ to a faulty word $v_{1}$ by repeating the cycle, while having this faulty word $v_{1} v_{2}$ equivalent to a safe one $v^{\prime}$. This proves non-diagnosability. The if part uses the finiteness of $A$, as a long enough suffix $v_{2}$ necessarily contains a (faulty) cycle of $B$ that can be matched to an observationally equivalent safe/normal cycle of $B$.

The original version of Proposition 1 actually relied on a twin machine directly built from $A$ and not from $B=\operatorname{Red}_{\Sigma_{o}}(A)$. Building a basis for cycles in a graph can be done in polynomial time w.r.t the size of the original graph [12]. As $C$ is at most 
of quadratic size w.r.t. the size of $A$, Proposition 1 clearly yields a polynomial test for the diagnosability of $A$.

\section{Diagnosability of repairable faults}

\subsection{Diagnosis and T-diagnosability}

We still consider a $\Sigma_{o}$-live deterministic automaton $A$, and now assume that some faults in $A$ can be repaired, i.e. $A$ contains transitions from $S_{F}$ to $S_{N}$, or equivalently that the fault language $L_{F}(A)$ is not saturated. The diagnosis of an observed sequence $w=\sigma_{o}(u)$ produced by some run $u$ of $A$ is defined as in (2). However, we reinforce the diagnosability criterion for $A$ by requiring that, when some fault occurs, it is still detected in finite time, but also before it is repaired.

Let us first introduce some notation. We denote by $L_{F}^{\min }=\left\{v \alpha \in L_{F}(A) \mid v \notin\right.$ $\left.L_{F}(A) \wedge \alpha \in \Sigma\right\}$ the set of minimal faulty words of $A$, i.e. words that correspond to a run ending with a transition from a normal state to a faulty one in $A$. For a word $v_{1} \in L_{F}(A)$, let $v_{1} \rightarrow v_{1} v_{2} \in L_{F}(A)$ denote the continuous presence of a fault along $v_{2}$. Formally, $v_{1} \rightarrow v_{1} v_{2} \in L_{F}(A)$ iff $\forall v_{2}^{\prime} \leq v_{2}, v_{1} v_{2}^{\prime} \in L_{F}(A)$, where $\leq$ denotes the prefix relation on words.

Formally, an automaton $A$ is timely diagnosable (T-diagnosable for short) iff

$$
\begin{gathered}
\forall v_{1} \in L_{F}^{\min }(A), \exists n \in \mathbb{N}, \forall v_{1} v_{2} \in L(A), \\
{\left[\left|v_{2}\right|_{o} \geq n \Rightarrow \exists v_{2}^{\prime} \leq v_{2}: v_{1} \rightarrow v_{1} v_{2}^{\prime} \in L_{F}(A)\right.} \\
\left.\wedge \Pi^{-1} \circ \Pi\left(v_{1} v_{2}^{\prime}\right) \subseteq L_{F}(A)\right]
\end{gathered}
$$

T-diagnosability differs from Def. (3) mainly by requiring that the fault that appears in $v_{1}$ remains for the whole execution of prefix $v_{2}^{\prime}$. This notion is illustrated in Fig. 3, that depicts several observationally equivalent runs, and shows observation times at which a correct diagnosis/detection can be produced (before repair). Observe that if faults are not repairable, $v_{1} \in L_{F}^{\min }(A)$ implies that $v_{1} \rightarrow v_{1} v_{2}^{\prime} \in L_{F}(A)$ for every $v_{2}^{\prime}$, and Def. (5) reduces to Def. (3) (condition $\forall v_{1} \in L_{F}(A)$ in Def. (3) can equivalently be replaced by $\left.\forall v_{1} \in L_{F}^{\min }(A)\right)$. So, in a setting of permanent faults, T-diagnosability is equivalent to diagnosability.

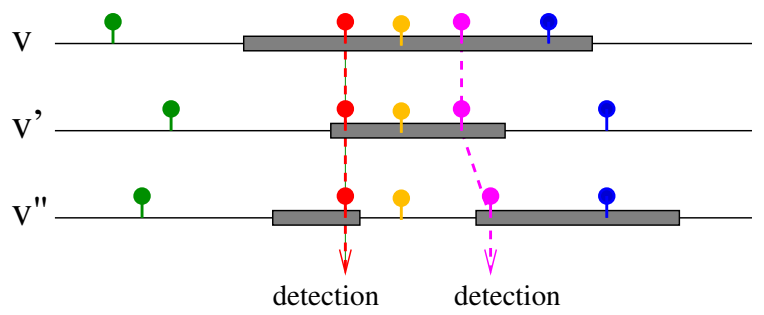

Fig. 3 A faulty word $v$ and two equivalent words $v^{\prime}, v^{\prime \prime}$. The observed labels are represented as pins, and the faulty zones as grey rectangles. Detections correspond to times (in number of observations) where all equivalent words are faulty. 
Fig. 4 illustrates the notion of T-diagnosability. Safe (resp. faulty) states are represented as circles (resp. boxes) One has $\Sigma=\{a, b, c, d\}$ and $\Sigma_{o}=\{a\}$. Ignoring the dashed transitions at the bottom, the automaton is T-diagnosable as after the observation of sequence $a$ a fault occurred in both runs at the top, and this fault is each time detected before it is repaired since $\Delta(a)=F$. By adding the bottom part, Tdiagnosability is lost: once $a$ has been observed, one knows for sure that a fault occurred, but no detection can take place before repair, in all runs, as now $\Delta(a)=U$.

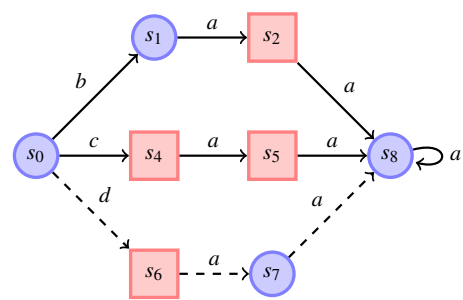

Fig. 4 A T-diagnosable system, when the path at the bottom is ignored.

\subsection{Vanishing faults and repairs}

T-diagnosability seems to be a reasonable first step towards the ability to count fault occurrences. Unfortunately, this is not the case as it is already apparent in Fig. 3 : an automaton with such equivalent runs can be T-diagnosable, and nevertheless the same observed sequence matches a run with one fault (top) and one with two faults (bottom). The situation is even worse. Let us call a vanishing fault a fault that occurs and is repaired in the silent part of a run of $A$ (i.e. between two observations), and similarly let us call a vanishing repair a transition from a faulty state to a safe one that occurs between two observations.

An automaton $A$ can exhibit runs with an arbitrary number of vanishing faults and repairs without losing its T-diagnosability. This is illustrated by the example in Fig. 5, with $\Sigma=\{a, b\}, \Sigma_{o}=\{a\}$. In this automaton $A$, one has $\Delta(a)=F$. A vanishing repair appears at the end of word $a b$ and a vanishing fault at the end of $a b b$. Nevertheless, Tdiagnosability holds : for $v_{1}=a \in L_{F}^{\min }(A)$ one gets immediate fault detection $\left(v_{2}=\varepsilon\right.$ works), for $v_{1}=a b^{2} \in L_{F}^{\min }(A)$ one has $\Pi^{-1}\left(\Pi\left(v_{1}\right)\right)=\{a\} \subseteq L_{F}(A)$ so again the fault detection is "immediate" with $v_{2}=\varepsilon$, and similarly for $v_{1}=a b^{4} \in L_{F}^{\min }(A)$.

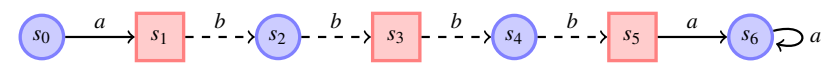

Fig. 5 An arbitrary number of vanishing faults and repairs may exist in a T-diagnosable automaton.

It is quite counter-intuitive that the "immediate" detection of the fault occurring at $v_{1}=a b^{2}$ actually relies on the detection of the fault that took place previously, at $v_{1}=$ 
$a$. This phenomenon is due to the fact that T-diagnosability, just as diagnosability, only refers to runs that stop at a visible transition. Everything that happens between observations is almost ignored. For permanent faults, this is harmless: it only shifts the detection by one observation. For repairable faults, it introduces odd phenomena like the ones mentioned above. A natural way to make fault detection causal (and to open the way to a counting of faults) is thus to forbid the existence of vanishing repairs

$$
\begin{gathered}
\nexists v=v_{1} v_{2} \alpha \in L(A): v_{1} \in L_{F}(A) \wedge v_{1} v_{2} \in L_{N}(A) \\
\wedge \alpha \in \Sigma \wedge v_{1} v_{2} \alpha \in L_{F}(A) \wedge \Pi\left(v_{2}\right)=\varepsilon
\end{gathered}
$$

and of vanishing faults

$$
\begin{gathered}
\nexists v=v_{1} v_{2} \alpha \in L(A): v_{1} \in L_{N}(A) \wedge v_{1} v_{2} \in L_{F}(A) \\
\wedge \alpha \in \Sigma \wedge v_{1} v_{2} \alpha \in L_{N}(A) \wedge \Pi\left(v_{2}\right)=\varepsilon
\end{gathered}
$$

Under these assumptions, at most one transition from $S_{F}$ to $S_{N}$ or from $S_{N}$ to $S_{F}$ can take place between two visible events. Let us say that fault detection is causal when observations following the fault enable its detection, so the detection does not depend on observations that occurred strictly before the fault as in the pathological cases above. Such causality can then be expressed as follows.

Proposition 2 Assuming (6) and (7), A is T-diagnosable if and only if

$$
\begin{gathered}
\forall v_{1} \in L_{F, o}^{\min }(A) \cup L_{F, u}^{\min }(A) \Sigma_{u}^{*} \Sigma_{o}, \exists n \in \mathbb{N}, \forall v_{1} v_{2} \in L(A), \\
{\left[\left|v_{2}\right|_{o} \geq n \Rightarrow \exists v_{2}^{\prime} \leq v_{2}: v_{1} \rightarrow v_{1} v_{2}^{\prime} \in L_{F}(A)\right.} \\
\left.\wedge v_{1} v_{2}^{\prime} \in \Sigma^{*} \Sigma_{o} \wedge \Pi^{-1} \circ \Pi\left(v_{1} v_{2}^{\prime}\right) \subseteq L_{F}(A)\right]
\end{gathered}
$$

where $L_{F, o}^{\min }(A)=L_{F}^{\min }(A) \cap \Sigma^{*} \Sigma_{o}$ represent minimal faulty runs that terminate with a visible event, and $L_{F, u}^{\min }(A)=L_{F}^{\min }(A) \backslash L_{F, o}^{\min }(A)$ represent those that terminate with a silent event.

Proof The extra condition $v_{1} v_{2}^{\prime} \in \Sigma^{*} \Sigma_{o}$ requires that the fault detection takes place at the moment one gets an observation. This could have been introduced in (5) without loss of generality, as silent events at the end of $v_{2}^{\prime}$ are useless to the criterion $\Pi^{-1}$ 。 $\Pi\left(v_{1} v_{2}^{\prime}\right) \subseteq L_{F}(A)$. So the only novelty lies in the first term. Recall that $L_{F}^{\min }(A)=$ $L_{F, o}^{\min }(A) \uplus L_{F, u}^{\min }(A)$. Words $v_{1} \in L_{F, o}^{\min }(A)$ are considered by both (5) and (8). But words $v_{1} \in L_{F, u}^{\min }(A)$ in (5) are replaced by words $v_{1} \in L_{F, u}^{\min }(A) \Sigma_{u}^{*} \Sigma_{o}$ in (8). In other words, for faults that occur silently, detection takes places after the next visible event.

Only if part. Assume $A$ is T-diagnosable, and let $v_{1} \in L_{F, u}^{\min }(A) \Sigma_{u}^{*} \Sigma_{o} . v_{1}$ decomposes uniquely as $v_{1}=v_{0} u_{3}$ where $v_{0}$ is the $L_{F, u}^{\min }$ part and $u_{3}$ the extension in $\Sigma_{u}^{*} \Sigma_{o}$. $v_{0}$ further decomposes as $v_{0}=u_{1} u_{2}$ where $u_{2}$ is the longest silent suffix of $v_{0}$. Thanks to (6) and (7), one has that $u_{1} \in L_{N}(A)$, and $u_{1} u_{2} \rightarrow u_{1} u_{2} u_{3} \in L_{F}(A)$. As $A$ is Tdiagnosable and $v_{0} \in L_{F}^{\min }(A)$, let us take any long enough extension $v_{2} \geq u_{3}$ for the fault detection in Def. (5), and let $v_{2}^{\prime} \leq v_{2}, v_{2}^{\prime} \in \Sigma^{*} \Sigma_{o}$ be the detection time. One can not have $v_{2}^{\prime}<u_{3}$ because in that case $\Pi\left(v_{0} v_{2}^{\prime}\right)=\Pi\left(v_{0}\right)=\Pi\left(u_{1}\right)$ and $u_{1} \in L_{N}(A)$. So the detection of the fault can not occur before the extra observation lying at the end 
of $u_{3}$. Since $v_{2}^{\prime} \geq u_{3}$, one has $v_{2}^{\prime}=u_{3} v_{2}^{\prime \prime}$ and $v_{0} \rightarrow v_{0} u_{3} v_{2}^{\prime \prime} \in L_{F}(A)$. This proves the existence of a detection time $v_{2}^{\prime \prime}$ after $v_{1}=v_{0} u_{3}$ which satisfies (8).

If part. Assume $A$ satisfies (8) and let $v_{1} \in L_{F, u}^{\min }(A) . v_{1}$ decomposes uniquely as $v_{1}=u_{1} u_{2}$ where $u_{2}$ is the longest silent suffix of $v_{1}$. Thanks to (6) and (7), one has $v_{1} \in L_{N}(A)$. Let $v_{1} v_{2} \in L(A)$, with $v_{2}$ long enough, in particular $\left|v_{2}\right|_{o} \geq 1$. One can write $v_{2}=u_{3} u_{4}$ with $u_{3} \in \Sigma_{u}^{*} \Sigma_{o}$. Thanks to (6) and (7) again, one has $v_{1} \rightarrow v_{1} u_{3} \in$ $L_{F}(A)$. As $v_{1} u_{3} \in L_{F, u}^{\min }(A) \Sigma_{u}^{*} \Sigma_{o}$ and $u_{4}$ is long enough, there exists a prefix $u_{4}^{\prime} \leq u_{4}$ such that $v_{1} u_{3} \rightarrow v_{1} u_{3} u_{4}^{\prime} \in L_{F}(A)$ and $\Delta\left(\Pi\left(v_{1} u_{3} u_{4}^{\prime}\right)\right)=F$. Taking $v_{2}^{\prime}=u_{3} u_{4}^{\prime}$ thus satisfies the conditions of (5).

Vanishing faults (or repairs) can be considered as design errors in system $A$, that are either benign and should be disregarded, or conversely that are possibly harmful and should be made visible. Changing the status of such events means modifyng the safe and faulty words of $A$, which is doable (see later). Meanwhile we show that the detection of vanishing faults/repairs is a simple problem: these events are regular properties along a run, and they can thus be evidenced by standard state augmentation techniques. The construction is given below.

Proposition 3 Detecting whether an automaton has vanishing faults (resp. repairs) can be done in NLOGSPACE, and in linear time wrt to the size of A.

Proof An automaton $A$ can produce a vanishing fault iff it contains an accessible run $u=t_{1} \cdot t_{2} \ldots t_{k}$ such that $s^{-}\left(t_{i}\right) \in S_{F}$ for every $i \in 2 \ldots k, s^{-}\left(t_{1}\right), s^{+}\left(t_{k}\right) \in S_{N}$, and $\sigma\left(t_{1} \ldots t_{k-1}\right)=\varepsilon$ (the last transition $t_{k}$ can be observable or not). Such run will be called a vanishing fault sequence.

Starting from a automaton $A$ one can build a non-deterministic automaton $V A=$ $\left(S \times V, T^{\prime}, \Sigma,\left(s_{0}, N O\right)\right)$ where $V=\{N O, U F, V a n\}$ is a label with the following meaning : a state labeled by $N O$ is a state encountered along a run in which the decision to recognize a vanishing fault was not yet taken. A state labeled by $U F$ is a state that is encountered along a run in which a transition from a non-faulty state to a faulty one was met, and no observable action was observed since this transition. A state labeled by Van is a state appearing along a run in which a vanishing fault was found. The transition relation $T^{\prime}$ is defined as follows:

- $\left((s, N O), \sigma,\left(s^{\prime}, N O\right)\right) \in T^{\prime}$ if $\left(s, \sigma, s^{\prime}\right) \in T$. Note that even if $s$ is a non-faulty state and $s^{\prime}$ a faulty one, the automaton is not forced to recognize a vanishing sequence from this transition.

- $\left((s, N O), \sigma,\left(s^{\prime}, U F\right)\right) \in T^{\prime}$ if $\left(s, \sigma, s^{\prime}\right) \in T, \sigma \in \Sigma_{u}, s \in S_{N}, s^{\prime} \in S_{F}$. This transition is where recognition of a potential vanishing sequence starts.

- $\left((s, U F), \sigma,\left(s^{\prime}, U F\right)\right) \in T^{\prime}$ if $\left(s, \sigma, s^{\prime}\right) \in T, \sigma \in \Sigma_{u}, s \in S_{F}, s^{\prime} \in S_{F}$. A vanishing sequence recognition has started, and as long as the system remains faulty and does not produce observable actions, the currently followed run can be a vanishing sequence.

- $\left((s, U F), \sigma,\left(s^{\prime}\right.\right.$, Van $\left.)\right) \in T^{\prime}$ if $\left(s, \sigma, s^{\prime}\right) \in T, s \in S_{F}, s^{\prime} \in S_{N}$. Label $\sigma$ can be observable or not. This transition occurs as soon as a vanishing sequence is detected: the system was faulty and unobservable from the occurrence of a fault up to the occurrence of the repair. 
- $\left((s, U F), \sigma,\left(s^{\prime}, N O\right)\right) \in T^{\prime}$ if $\left(s, \sigma, s^{\prime}\right) \in T, \sigma \in \Sigma_{o}, s \in S_{F}$. An observable transition was reached that was not a repair, so the last fault was not a vanishing fault.

Obviously, automaton $A$ contains a vanishing sequence iff there exists a reachable state of the form $(s, \mathrm{Van})$ in $V A$. The size of $V A$ is at most 3.|A|, so $V A$ (restricted to its reachable states) can be built in $O(3 .|A|)$, and the search for a vanishing sequence can be performed non-deterministically using a standard reachability algorithm in logarithmic space (in the size of $|A|$ ), and if all states have to be explored, in time in $O(3 .|A|)$.

Figure 6 illustrates the construction of an automaton that recognizes vanishing sequences. The top sequence starts recognizing a sequence of unobserved action after a fault, but action $\sigma_{k}$ is observable, so the tag attached to the last state reached by the sequence gets back to $N O$. In the bottom sequence, the system returns to a normal state after observation of the empty word: a vanishing sequence has been detected. A similar construction can be used to detect vanishing repairs, just by moving from a $N O$ state to an $U F$ state as soon as an unobservable repair occurs, and from an $U F$ state to a Van state when a new unobservable fault occurs.

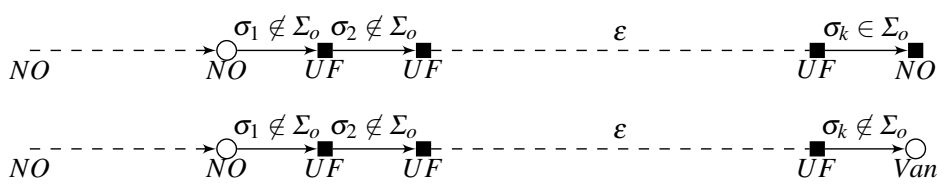

Fig. 6 Construction of automaton $V A$ to detect vanishing faults.

In the rest of the paper, we will assume that the considered systems do not contain vanishing faults (repairs). This assumption is not too constraining: a vanishing fault (repair) might not be followed by an observation, hence hindering any chance to diagnose it. If one want to diagnose fault in a timely way, the assumption that something observable necessarily occurs while the system is faulty is needed. We shall come back to this point later in the paper.

\subsection{A T-diagnosability test}

As in Section 2.3, one can consider the converse of (5). Specifically, $A$ is not $T$ diagnosable iff

$$
\begin{gathered}
\exists v_{1} \in L_{F}^{\min }(A), \forall n \in \mathbb{N}, \exists v_{1} v_{2} \in L(A):\left|v_{2}\right|_{o} \geq n, \\
\forall v_{2}^{\prime} \leq v_{2}, v_{1} \rightarrow v_{1} v_{2}^{\prime} \notin L_{F}(A) \vee \Pi^{-1} \circ \Pi\left(v_{1} v_{2}^{\prime}\right) \not \subset L_{F}(A)
\end{gathered}
$$

In words, $A$ is not T-diagnosable whenever it is possible to find a minimal faulty sequence $v_{1}$ and arbitrarily long extensions $v_{2}$ such that along the longest faulty prefix $v_{2}^{\prime} \leq v_{2}$ of $v_{2}$, the detection of the fault can not occur in a timely way, either because repair occurs before any possible detection, or because the extension remains ambiguous. 
It is worth noticing that the twin-machine idea used to check the diagnosability of permanent faults is not sufficient to check the T-diagnosability of repairable faults. The main obstacle comes from the fact that T-diagnosability can not be characterized by pairs of equivalent runs. It is rather a global property on classes of equivalent runs in $A$. This is illustrated in Fig. 7, where unobservable transitions are depicted as dashed arrows $\left(\Sigma_{o}=\{a\}\right)$ and faulty states in red. This automaton is not T-diagnosable. However, the twin machine built for this system in Fig. 8 contains no ambiguous cycle. By checking only pairs of equivalent runs, one always finds a time where ambiguity disappears. For example, considering only the top and central loops, $a^{3 n+1}$ seem to be detection times for the faults that appear in these runs. To reveal that T-diagnosability does not hold, one would have to check triples of equivalent runs here. And it is quite easy to design examples where triples are not sufficient and one needs to escalate to quadruples of equivalent runs to reveal the non T-diagnosability, etc. This suggests a non polynomial complexity of the T-diagnosability test.

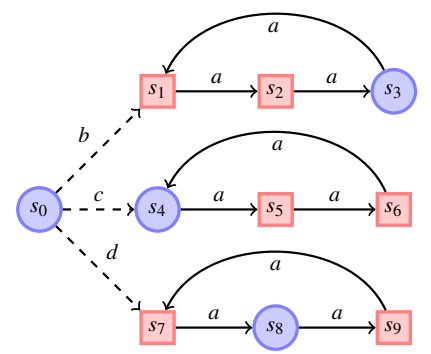

Fig. 7 A system that is not T-diagnosable.Considering only pairs of equivalent runs is not sufficient to characterize non-T-diagnosability.

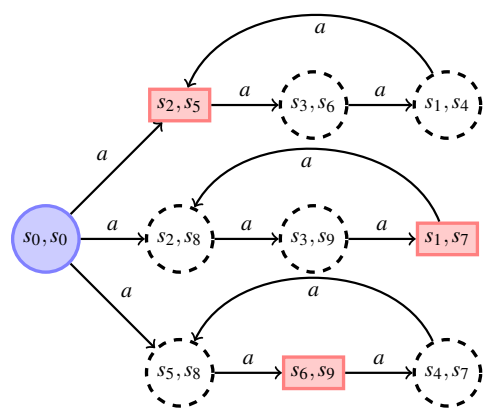

Fig. 8 The twin machine for the system of Fig. 7. Square states represent states of the twim machine with faulty pairs of states. Filled circle are states with non-faulty components, and dashed states are ambiguous states, i.e. in which one component is faulty and the other is safe. Every cycle of this twin machine contains a square faulty state. Though this system does not contain ambiguous cycles, the system is not T-diagnosable. 
The idea of the twin-machine construction is to check whether a faulty run can create an ambiguity that can never be resolved. For repairable faults, this ambiguity signal can be directly derived from $\operatorname{Diag}(A)$, the diagnoser of $A$. Consider the (deterministic) automaton $G=A \times \operatorname{Diag}(A)$. $\operatorname{Diag}(A)$ is a deterministic automaton over alphabet $\Sigma_{o} \subseteq \Sigma$, and $L(\operatorname{Diag}(A))=L_{o}(A)$. So $L(G)=L(A)$ : the construction of $G$ performs a simple state augmentation on $A$, without changing its behavior (just like the memory automaton mentioned above). This state augmentation attaches an ambiguity status to each state of $A$ as follows. States of $G$ take the form $(s, q) \in S \times Q$ where $Q=2^{S}$. So they can be labeled by elements in $\{N, F\} \times\{N, U, F\}$ : for example $(s, q)$ is of type $(N, U)$ iff $s \in S_{N}$ and $q$ is uncertain. $L_{N}(A)$ and $L_{F}(A)$ are easily identifiable in $G$ as words terminating in a state of type $(N,$.$) or (F,$.$) respectively. A$ state $(s, q)$ is said to be minimally faulty iff $s$ is the terminal state of a run $v_{1} \in L_{F}^{\min }(A)$. Notice that a state $(s, q)$ labeled $(F, N)$ can exist : it characterizes a run that was safe up to the last observation and that later produced (silently) a fault, no yet detectable that the diagnoser. Similarly, as state $(s, q)$ labeled $(F, U)$ characterizes a faulty run that is not yet diagnosed.

Theorem 1 With notation above, $A$ is not T-diagnosable if and only if there exists a reachable minimally faulty state $(s, q) \in S \times Q$ in $G$ such that $(s, q)$ is of type $(F, N)$ or $(F, U)$ and either

1. there exists a state $\left(s^{\prime}, q^{\prime}\right)$ of type $(N, N)$ or $(N, U)$

2. or there exists a cycle of $(F, U)$ states

that is reachable from $(s, q)$ through a (possibly empty) sequence of $(F, N)$ states followed by a sequence of $(F, U)$ states.

Proof By construction of $G$, observe that if word $v \in L(A)$ reaches state $s$ in $A$, then word $v$ reaches state $(s, q)$ in $G$ and $\Delta(\Pi(v))$ is the type of state $q \in Q$, either $N, F$ or $U$.

For the only if part, consider the witness $v_{1} \in L_{F}^{\min }(A)$ of non T-diagnosability in (9), which reaches state $(s, q)$ in $G .(s, q)$ is necessarily of type $(F, N)$ or of type $(F, U)$, as if $(s, q)$ is of type $(F, F)$ then the correct diagnosis is output with $v_{2}^{\prime}=\varepsilon$. For a given $n$, let $v_{2}$ be the extension of $v_{1}$ satisfying (9), and let $v_{2}^{\prime}$ be the longest prefix of $v_{2}$ such that $v_{1} \rightarrow v_{1} v_{2}^{\prime} \in L_{F}(A)$. All along $v_{2}^{\prime}$, the correct diagnosis can not be output, so $G$ only crosses states of type $(F, N)$ or $(F, U)$. States of type $(F, N)$ come first (if they exist), then (after the first observable event in $v_{2}^{\prime}$ ) one only crosses states of type $(F, U)$ as at least one faulty run lies in the inverse projection. If there exists $a \in \Sigma$ such that $v_{2}^{\prime} a \leq v_{2}$, then $v_{1} v_{2}^{\prime} a$ reaches state $\left(s^{\prime}, q^{\prime}\right)$ which is either of type $(N, N)$ or of type $(N, U) .(N, F)$ is not possible as this would mean that the correct diagnosis was produced for $v_{1} v_{2}^{\prime}$. This makes point 1 in the theorem. If point 1 never occurs for any $n$, this means that in the discussion above one always has $v_{2}^{\prime}=v_{2}$. As $G$ is finite, it then contains a cycle with at least one observable event (recall that $n$ counts observations). This cycle is thus made of $(F, U)$ states, which makes point 2 in the theorem.

The if part can be derived in a similar manner, starting from conditions in the theorem and building a witness $v_{1}$ and the associated $v_{2}$ for every $n$ satisfying (9). 
Back to the example depicted in Figure 7, the automaton $G=A \times \operatorname{Diag}(A)$ is given in Figure 9 (left-hand side), whereas its abstract view carrying only labels of composite states is given in Figure 9 (right-hand side) (recall that $\{b, c, d\}$ are unobservable). It is easy to check that $G$ does not fulfill the conditions of Theorem 1 . Indeed, $G$ contains a $(F, U)$ state, from which a $(N, U)$ state is reachable (this is highlighted in the figure by a dashed arrow). Thus, as already mentioned, $A$ is not T-diagnosable.
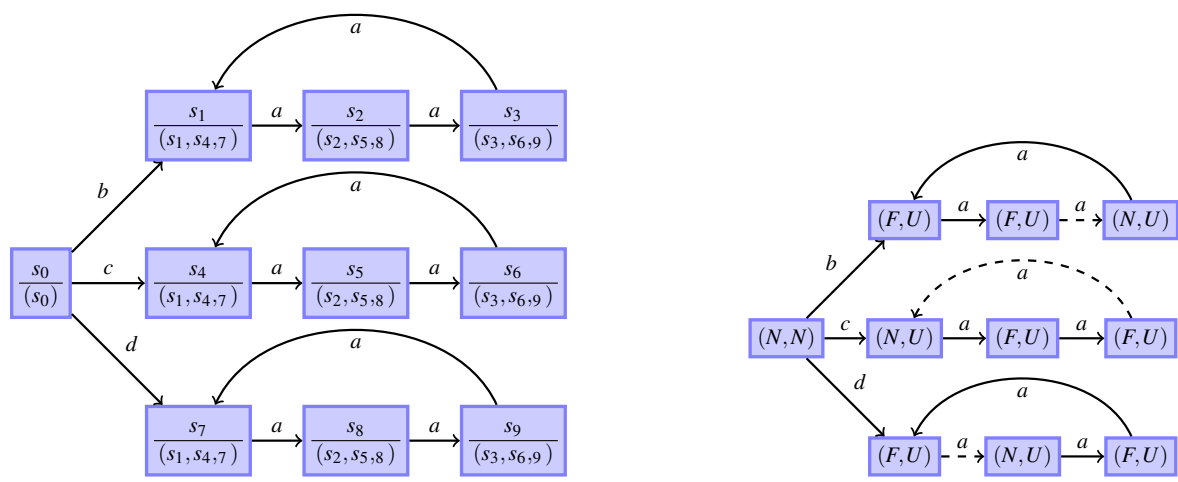

Fig. 9 The augmented machine $G=A \times \operatorname{Diag}(A)$ (left) and its abstract version (right), for the example given in FIg. 7.

\subsection{Complexity of T-diagnosability}

Theorem 2 Deciding whether an automaton A is T-diagnosable is a PSPACE-complete problem.

Proof First, we can easily show that T-diagnosability belongs to PSPACE. Following the result of Theorem 1, $A$ is not T-diagnosable iff one can find a witness cycle of type $(F, U)$ or a witness state of type $(N, N)$ or $(N, U)$ reachable after a minimally faulty sequence ending in a state of type $(F, N)$ or $(F, U)$ in $G$. First of all, the size of $G$ is at most $2^{|A|} .|A|$. To witness a minimally faulty sequence ending in a $(F, N)$ of $(F, U)$ state, one only needs to non-deterministically explore paths of size smaller than $2^{|A|} .|A|$, which can be done with polynomial memory size (to remember current state and whether previous state is faulty). Then, to witness ambiguous cycles or moves to $(N, N)$ or $(N, U)$ states, one can again non-deterministically explore paths of $G$ of size smaller than $2^{|A|} .|A|$ with polynomial memory. Hence, finding witness paths for non-T-diagnosability is a NPSPACE process, and using Savitch's theorem [11], and remembering that PSPACE is closed under complementation, this shows that T-diagnosability is in PSPACE.

The second step of the proof shows hardness of the problem by reduction from a language inclusion problem, which is known to be PSPACE-complete (see [8] and 


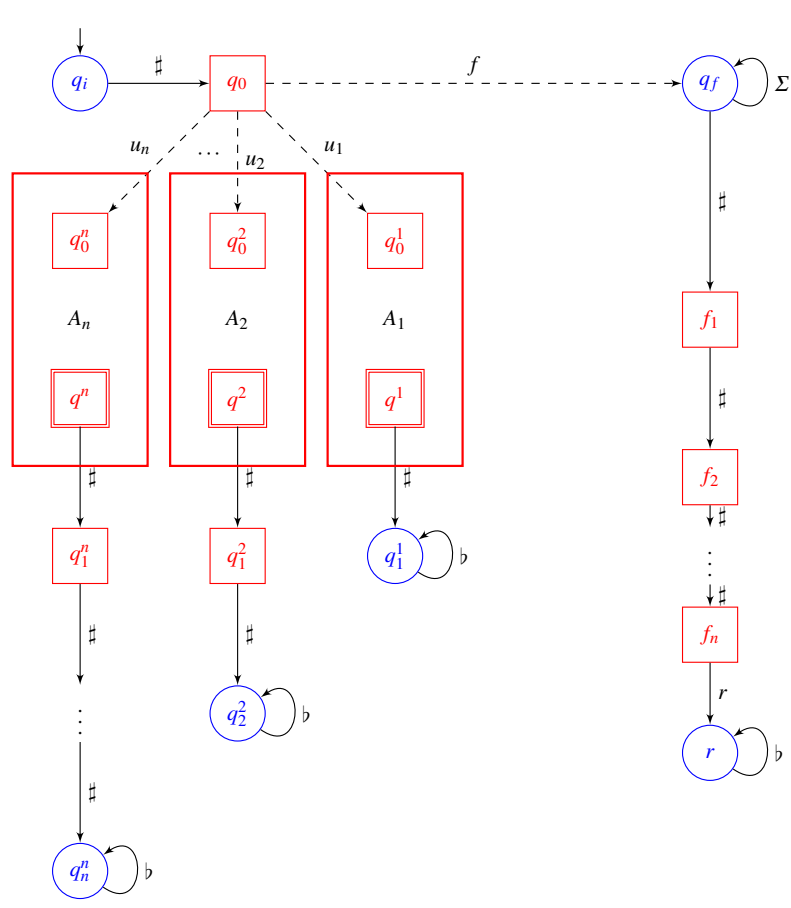

Fig. 10 PSPACE-hardness of T-diagnosability. Red states are faulty, and blue states normal states.

[9]). The problem can be formulated as follows: given $A_{1}, \ldots, A_{n}$ some deterministic finite automata, does $\bigcap_{i \in 1 . . n} L\left(A_{i}\right)=\emptyset$ ?

Let $n \in \mathbb{N}$ and for $1 \leq i \leq n, A_{i}=\left(S_{i}, \Gamma, T_{i}, q_{0}^{i}, F_{i}\right)$ be some deterministic finite automaton on alphabet $\Gamma$, that recognizes language $L\left(A_{i}\right)=\left\{\sigma(u) \mid s^{-}(u)=q_{0}^{i} \wedge\right.$ $\left.s^{+}(u) \in F_{i}\right\}$. We build the finite automaton $A=\left(S, \Sigma, T, q_{0}\right)$ (see Figure 10) where:

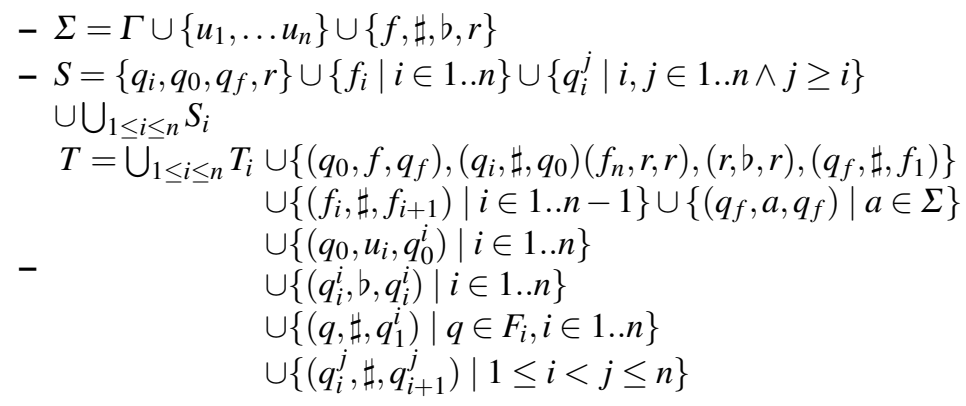

The set of safe states is $S_{N}=\left\{q_{i}, q_{f}, r\right\} \cup\left\{q_{i}^{i} \mid i=1 \ldots n\right\}$. The set of faulty states is $S \backslash S_{N}$. We set $\Sigma_{o}=\Gamma \cup\{\sharp, b, r\}$.

We claim that $A$ is T-diagnosable if and only if $\bigcap_{i \in 1 . . n} L\left(A_{i}\right)=\emptyset$.

First, remark that after observing $\sharp w \sharp^{m}$ for $m \leq n$, the current run is either in state $f_{m}$ or in some state $q_{m}^{j}$ if $j \geq m$, and automaton $A_{j}$ accepts $w$. 
Suppose that $A$ is T-diagnosable. Let $w \in \Sigma^{*}$, and let $v_{1}$ be the unique run of $A$ such that $\sigma\left(v_{1}\right)=\sharp f w \sharp$. As $v_{1}$ is a minimal faulty run, there exists $m \leq n$ such that the run $v_{1} v_{2}$ with $\sigma\left(v_{1} v_{2}\right)=\sharp f w \sharp^{m}$ verifies $\Pi^{-1} \circ \Pi\left(v_{1} v_{2}\right) \subseteq L_{F}(A)$.

According to the construction of $A$, if automaton $A_{i}$ accepts $w$, then for $i \geq m$, there exists also a run of $A$ which observation is $\sharp w \sharp^{m}$, and that ends in state $q_{m}^{i}$. Furtermore, as $\Pi^{-1} \circ \Pi\left(v_{1} v_{2}\right) \subseteq L_{F}(A)$, the system can not be in state $q_{m}^{m}$ as this state is safe. Hence, $A_{m}$ does not accept $w$ and $w$ does not belong to the intersection $\bigcap_{i \in 1 . . n} L\left(A_{i}\right)$. As this is true for every $w \in \Sigma^{*}$, T-diagosability of $A$ implies $\bigcap_{i \in 1 . . .} L\left(A_{i}\right)=\emptyset$.

Conversely suppose that $\bigcap_{i \in 1 . . n} L\left(A_{i}\right)=\emptyset$. Let $v_{1}$ be a minimal faulty run. Only two cases can appear: either $v_{1}$ is the word $v_{1}=\sharp$ which ends in the faulty state $q_{0}$, or $v_{1}$ is of the form $v_{1}=\sharp f w \sharp$. If $v_{1}=\sharp$, then we know that $A$ is in $q_{0}$ which is faulty and we can claim the fault. In the second case $s^{+}\left(v_{1}\right)=f_{1}$ and $\sigma\left(v_{1}\right)=\sharp f w \sharp$ with $w \in \Sigma^{*}$. As $\bigcap_{i \in 1 . . n}\left(A_{i}\right)=\emptyset$, there exists $i \in 1 . . n$ such that $w \notin L\left(A_{i}\right)$. Consider the run $v_{1} v_{2}$ with $\sigma\left(v_{1} v_{2}\right)=\sharp f w \sharp i$, this run ends in $f_{i}$ and was not repaired in between. Even if $w$ is recognized by $A_{k}$ for some $k<i$, no run visiting a state of $A_{k}$ and with observation $w \sharp i$ exists. Moreover, for every $j>i$ such that $A_{j}$ accepts $w$, runs with the same observation $w \sharp^{i}$ ends in state $q_{i}^{j}$. As $A_{i}$ does not accept $w$ and as all states $q_{i}^{j}$ for $j>i$ are faulty, $\Pi^{-1} \circ \Pi\left(v_{1} v_{2}\right) \in L_{F}(A)$. Thus the fault can be claimed. As this is true for every minimal faulty run $v_{1}, A$ is T-diagnosable.

\subsection{Removing the vanishing fault hypothesis}

It is worth noticing that the verification of the T-diagnosability assumes absence of vanishing faults (resp. repairs). However, as mentioned at the end of section 3.2, whenever there exist a vanishing fault in the system, the diagnoser is not able to detect it, as its verdict is given according to the occurrence of observable events. Thus, if one wants to diagnose fault in a timely way, the assumption that something observable necessarily occurs while the system is faulty is needed. An easy way to guarantee this property is to reduce the unobservable part of the alphabet. Obviously, if $\Sigma_{o}=\Sigma$, there can be no vanishing fault, but finer modifications of the original systems exist. For instance one can easily reuse the paths of automaton $V A$ leading from a fault to a vanishing state to discover the smallest subsets of unobservable actions that should become observable to avoid vanishing faults (the method would be similar to the sensor minimization technique proposed in [2] which aims at minimizing the size of observable events while keeping a system diagnosable).

However, un-observability of some actions is not always a design choice. In a distributed system, for instance, one may observe actions on a limited subset of machines. Another way to avoid vanishing faults is to consider that vanishing and hence potentially fully unobservable faults are not important failures, but rather design errors producing specification where the incriminated state change leading to a vanishing fault should not be considered as a fault. So, the next question to study is: how to transform a specification $A$ with vanishing faults into a correct specification $A^{\prime}$ without vanishing faults such that the detectability status of the non vanishing fault in $A$ are preserved in $A^{\prime}$ ? 
Let $A=\left(S, \Sigma, T, s_{0}\right)$ be a deterministic automaton, and $V A$ be the automaton built in proof of proposition 3 to detect sequences that contain a vanishing fault. We denote by $L_{V a n}(A)$ the language of vanishing faults, i.e. the set of words from $\Sigma^{*}$ that correspond to sequences with a vanishing fault. Formally,

$$
\begin{gathered}
L_{\operatorname{Van}}(A)=\left\{w \in \Sigma^{*} \mid \exists t_{1} \ldots t_{k} \in V A, s^{-}\left(t_{1}\right)=\left(s_{0}, N O\right), s^{+}\left(t_{k}\right) \in S \times\{\operatorname{Van}\},\right. \\
\left.\sigma\left(t_{1} \ldots t_{k}\right)=w\right\} .
\end{gathered}
$$

To ignore vanishing faults, we use a construction similar to that of automaton $V A$. our aim is to design an automaton $A^{\prime}$ such that $L(A)=L\left(A^{\prime}\right)$ and $L_{V a n}\left(A^{\prime}\right)=\emptyset$ and such that all the faulty sequences that can be diagnosed in $A$ are also diagnosed in $A^{\prime}$. We define the set of diagnosable sequences of $A$ as follows:

$$
\begin{aligned}
L_{\text {diag }}(A)=\left\{v_{1} \in L_{F}^{\min }(A) \mid\right. & \exists n \in \mathbb{N}, \forall v_{1} v_{2} \in L(A), \\
& {\left[\left|v_{2}\right|_{o} \geq n \Rightarrow \exists v_{2}^{\prime} \leq v_{2}: v_{1} \rightarrow v_{1} v_{2}^{\prime} \in L_{F}(A)\right.} \\
& \left.\left.\wedge \Pi^{-1} \circ \Pi\left(v_{1} v_{2}^{\prime}\right) \subseteq L_{F}(A)\right]\right\}
\end{aligned}
$$

Such an automaton is obtained as follows: we let $A^{\prime}=\left(S_{A^{\prime}}, \Sigma, T^{\prime},\left(s_{0}, N O\right)\right)$, where $S_{A^{\prime}}=S \times\{N O, U F, \operatorname{Van}\}^{2}$ and $T^{\prime}$ is built as follows:

- $\left((s, N O), \sigma,\left(s^{\prime}, N O\right)\right) \in T^{\prime}$ if $\left(s, \sigma, s^{\prime}\right) \in T$, and $\sigma \in \Sigma_{o}$

- $\left((s, N O), \sigma,\left(s^{\prime}, N O\right)\right) \in T^{\prime}$ if $\left(s, \sigma, s^{\prime}\right) \in T, \sigma \in \Sigma_{u o}$ and $s^{\prime} \in S_{N}$

- $\left((s, N O), \sigma,\left(s^{\prime}, N O\right)\right) \in T^{\prime}$ if $\left(s, \sigma, s^{\prime}\right) \in T$, and $\sigma \in \Sigma_{u o}, s \in S_{F}$ and $s^{\prime} \in S_{F}$

- $\left((s, N O), \sigma,\left(s^{\prime}, U F\right)\right) \in T^{\prime}$ if $\left(s, \sigma, s^{\prime}\right) \in T$, and $\sigma \in \Sigma_{u o}, s \in S_{N}$ and $s^{\prime} \in S_{F}$

- $\left((s, U F), \sigma,\left(s^{\prime}, U F\right)\right) \in T^{\prime}$ if $\left(s, \sigma, s^{\prime}\right) \in T$, and $\sigma \in \Sigma_{u o}$, and $s^{\prime} \in S_{F}$

- $\left((s, U F), \sigma,\left(s^{\prime}, N O\right)\right) \in T^{\prime}$ if $\left(s, \sigma, s^{\prime}\right) \in T$, and $\sigma \in \Sigma_{o}$ and $s^{\prime} \in S_{F}$

- $\left((s, U F), \sigma,\left(s^{\prime}\right.\right.$, Van $\left.)\right) \in T^{\prime}$ if $\left(s, \sigma, s^{\prime}\right) \in T$ and $s^{\prime} \in S_{N}$

Note that one necessarily has $s \in S_{F}$ in the three previous cases, as a fault has occurred (witnessed by label $U F$ ), and is not yet repaired.

- $\left((s\right.$, Van $\left.), \sigma,\left(s^{\prime}, N O\right)\right) \in T^{\prime}$ if $\left(s, \sigma, s^{\prime}\right) \in T$ and $\sigma \in \Sigma_{o}$.

- $\left((s\right.$, Van $\left.), \sigma,\left(s^{\prime}, U F\right)\right) \in T^{\prime}$ if $\left(s, \sigma, s^{\prime}\right) \in T, \sigma \in \Sigma_{u o}$ and $s^{\prime} \in S_{F}$.

- $\left((s\right.$, Van $\left.), \sigma,\left(s^{\prime}, N O\right)\right) \in T^{\prime}$ if $\left(s, \sigma, s^{\prime}\right) \in T, \sigma \in \Sigma_{u o}$ and $s^{\prime} \in S_{N}$.

Note that $s \in S_{N}$ in the three previous cases, as vanishing faults are detected only after a repair.

Let $\operatorname{CoaccVan}\left(A^{\prime}, X\right)$ denote the set of states of $A^{\prime}$ for which there exists a sequence of transitions that are all unobservable, excepted possibly the last transition, leading to a state of $X$. Then, we partition $S_{A^{\prime}}$ into $S_{N}^{\prime} \uplus S_{F}^{\prime}$, where $S_{F}^{\prime}=S_{F} \otimes\{U F, N O\} \backslash$ $\operatorname{CoaccVan}\left(A^{\prime}, S \otimes\{\operatorname{Van}\}\right.$, and $S_{N}^{\prime}=S^{\prime} \backslash S_{F}$. Informally, we consider as faulty the sets of states from which a fault will necessarily be followed by an observation before repair, i.e. it will not vanish.

\footnotetext{
2 where $N O, U F$, Van have the same meaning as in the automaton $V A$.
} 
Intuitively, in this construction, automaton $A^{\prime}$ memorizes if a fault has occurred, if an observable action has occurred since occurrence of a fault, and if a repair occurs before occurrence of an observable action.

We can now prove the following propositions:

Proposition $4 L\left(A^{\prime}\right)=L(A)$

Proof We can show $L\left(A^{\prime}\right) \subseteq L(A)$ and $L(A) \subseteq L\left(A^{\prime}\right)$ by induction on the length of words. Obviously, $\varepsilon \in L\left(A^{\prime}\right) \cap L(A)$. Let us now suppose that inclusion is verified in both directions for words of length at most $n$. Let $w$ be a words of $L(a)$ and $L\left(A^{\prime}\right)$. This word corresponds to a run of $A$ from $s_{0}$ to some state $s$, and to a run from $\left(s_{0}, N O\right)$ to a state $(s, l)$ where $l \in\{N O, U F, \operatorname{Van}\}$. Let us assume that $\left(s, \sigma, s^{\prime}\right) \in T$. According to the construction rules, if $\sigma \in \Sigma_{o}$ then regardless of label $l$, a transition labeled by $\sigma$ to some state $\left(s^{\prime}, l^{\prime}\right)$ exists. Conversely, if $w$ leads $A$ to some state $(s, l)$ and a transition $\left((s, l), \sigma,\left(s^{\prime}, l^{\prime}\right)\right)$ exists in $T^{\prime}$, then $\left(s, \sigma, s^{\prime}\right)$ is also a transition of $T$.

Proposition $5 L_{V a n}\left(A^{\prime}\right)=\emptyset$

Proof Let us assume that there exists a vanishing sequence in $A^{\prime}$. this sequence is of the form $u=t_{1} \ldots t_{n}$ such that $s^{-}\left(t_{1}\right)=\left(s_{0}, N O\right) \in S_{N}^{\prime}, s^{+}\left(t_{n}\right) \in S_{N}^{\prime}$, and there exists $k \in 1 . . n-1$ such that $\left(s_{k-1}, l_{k-1}\right)=s^{-}\left(t_{k}\right) \in S_{N}^{\prime},\left(s_{k}, l_{k}\right)=s^{+}\left(t_{k}\right) \in S_{F}^{\prime}, \sigma\left(t_{k} \ldots t_{n-1}\right)=$ $\varepsilon$, and $\sigma\left(t_{n}\right) \in \Sigma_{o}$. Let us first notice that $S_{F}^{\prime}$ is a subset of $S_{F} \times\{U F, N O\}$, and that the transition from $\left(s_{k-1}, l_{k-1}\right)$ to $\left(s_{k}, l_{k}\right)$ along the chosen sequence is unobservable. Hence, according to the construction rules for $A^{\prime}$, we have $l_{k}=U F$.

Similarly, one has $l_{i}=U F$ for every $i \in k+1 . . n-1$ as otherwise, the considered sequence $t_{k} \ldots t_{n-1}$ would be an observable one. Let us now consider $l_{n}$. As the last state reached by the sequence is a normal state, but as $l_{n-1}=U F$, we necessarily have $l_{n}=\operatorname{Van}$. This contradicts the rule imposed for the construction of $S_{F}^{\prime}$, that requires that no unobservable path leads from a faulty state in $S_{F}^{\prime}$ to a state with label Van . One can handle similarly the case where the last transition $t_{n}$ is not observable.

Let us illustrate the construction of $A^{\prime}$ on the example of Figure 11. In this automaton, $\Sigma_{o}=\left\{a, a^{\prime}\right\}, \Sigma_{u o}=\{b, c, d\}$. One can immediately notice that the run $v=\left(q_{0}, b, q_{2}\right)\left(q_{2}, b, q_{3}\right)\left(q_{3}, b, q_{5}\right)$ contains a vanishing fault. Figure 12 shown how the automaton $A$ is unfolded and how the resulting states are tagged wit $N O, U F, V a n$. Let us highlight the difference between $q_{2}, U F$ and $q_{2}, N O: q_{2}, N O$ identifies state $q_{2}$ of the system reached when no unobservable move from a non-faulty sate to a faulty one has been observed. Conversely, state $q_{2}, U F$ represents state $q_{2}$ of the system when an unobservable move from a safe state to a faulty one occurred. Black states in this figure are states of the form $(s, l)$ such that $s \in S_{F}$. However, the faulty states of $A^{\prime}$ consists in this subset of states without $\left(q_{2}, U F\right)$ and $\left(q_{3}, U F\right)$, that are in CoaccVan $\left(A^{\prime},\left\{\left(q_{5}, \operatorname{Van}\right\}\right)\right.$. The final automaton $A^{\prime}$ computed is depicted in Figure 13 We hence have $S_{F}^{\prime}=\left\{\left(q_{2}, N O\right),\left(q_{3}, N O\right),\left(q_{4}, N O\right),\left(q_{7}, U F\right),\left(q_{7}, N O\right)\right\}$. Remark that $L(A)=L\left(A^{\prime}\right)$ : both languages define the prefix closure of the regular language $b a^{*}+(b a+b) \cdot b \cdot\left(a b a^{*}+\right.$ $\left.b a^{*}\right)$. On this example, one can see that the construction simply adds memory to states of the system, to capture the information on whether a fault occurred, and whether an observable action followed or not. 


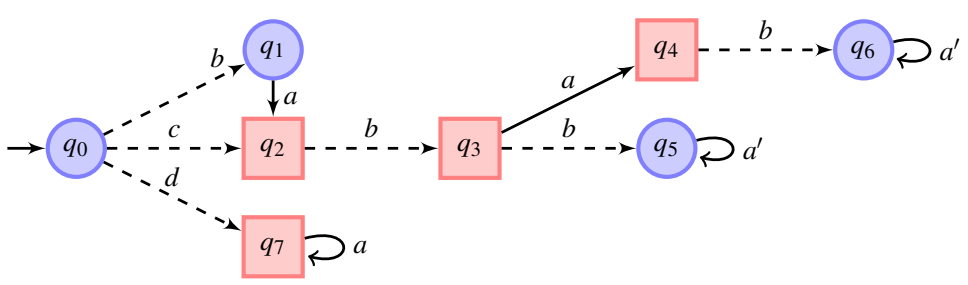

Fig. 11 An example of system with vanishing faults. $\Sigma_{O}=\{a\}, \Sigma_{u o}=\{b\}$.

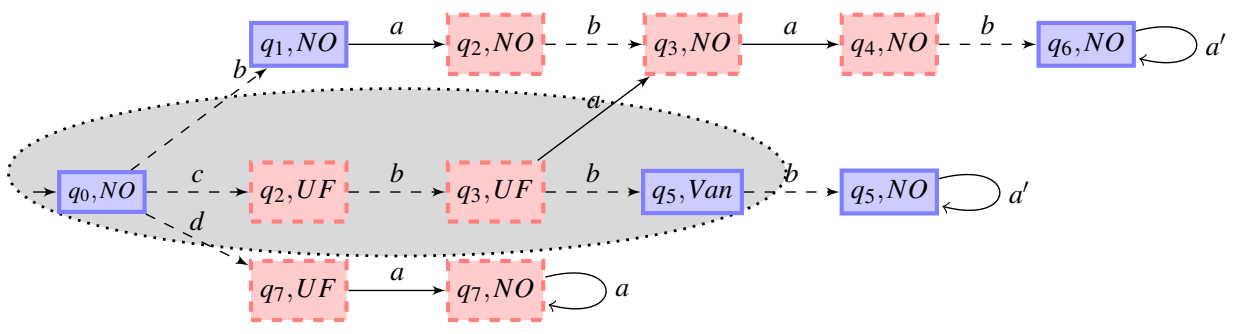

Fig. 12 Adding memory to the system of Figure 11. blue square states are states of the form $(s, l)$ where $s \in S_{F}$. red dashed square states are states of the form $(s, l)$ where $s \in S_{N}$. Note that all these states are not necessarily faulty in $A^{\prime}$. States in CoaccVan $\left(A^{\prime},\left\{\left(q_{5}, \operatorname{Van}\right)\right\}\right)$ are $\left\{\left(q_{0}, N O\right),\left(q_{2}, U F\right),\left(q_{3}, U F\right)\right\}$, and are represented in the dotted zone.

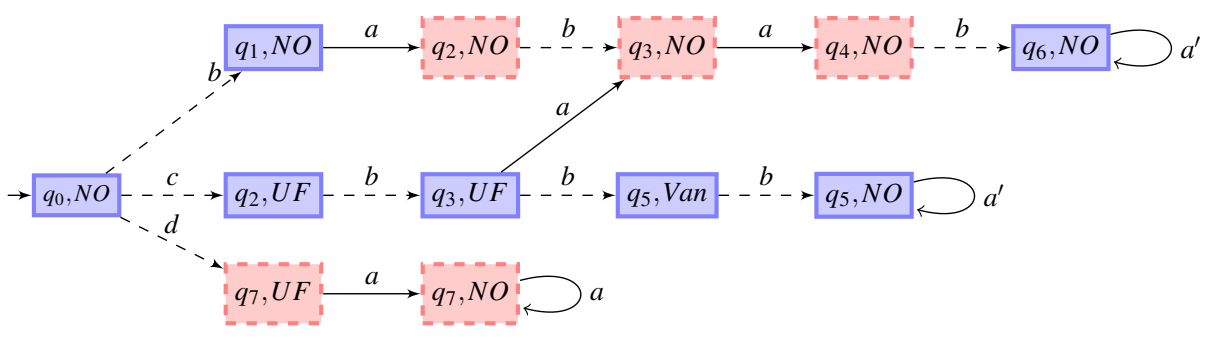

Fig. $13 \mathrm{~A}$ new automaton $A^{\prime}$ without vanishing faults. Faulty states are $S_{F}^{\prime}=$ $\left\{\left(q_{2}, N O\right),\left(q_{3}, N O\right),\left(q_{4}, N O\right),\left(q_{7}, U F\right),\left(q_{7}, N O\right)\right\}$.

The last question to address is how diagnosability of a system is affected by vanishing faults removal. Let us first recall that removing vanishing faults and repairs allows to get back to a setting where the characterization of diagnosability proposed in Theorem 1 applies, and also makes T-diagnosis causal. Note however that removing vanishing faults does not make a system diagnosable: Indeed, even if all faults are eventually followed by an observation before they are repaired, this does not mean that all ambiguity in a system is resolved, and several faults may remain ambiguous forever. A desirable property is that all faults that were in $L_{\text {diag }}(A)$ remain in $L_{\text {diag }}\left(A^{\prime}\right)$. For example, this is the case in Figure 13. Indeed, for this example, we have : $L_{F}^{\min }(A)=\{b a, c, d\}, L_{F}^{\min }\left(A^{\prime}\right)=\{b a, c b a, d\}, L_{\text {diag }}(A)=\{b a\}$, and $L_{\text {diag }}\left(A^{\prime}\right)=\{b a, c b a, d\}$. Knowing whether this property holds systematically is still an open problem. 


\section{Counting faults}

As faults are not permanent, counting the number of faults occurring at runtime is a useful information: even if a system is able to repair all occurrences of faults, a too large number of faults may indicate a major failure. To count faults, an immediate idea is to maintain a fault counter that is incremented each time the diagnoser goes from $N$ to $F$ and from $U$ to $F$. Even if a diagnosis can be triggered in time, i.e. before the fault is repaired, T-diagnosability is not sufficient to correctly count faults along a trajectory. Fig. 3 reveals that this can not work as counting moves of the diagnoser from $\{N, U\}$ to $F$ in this example would detect two faults, while $v$ has only one fault and $v^{\prime \prime}$ has two. Conversely, counting only moves from $N$ to $F$ or from $U$ to $F$ leads to minoring the real number of faults that occurred in some runs. This section considers extra conditions that enable counting. A fault counter $C$ of an automaton $A$ is a function from $\Sigma_{o}^{*}$ to $\mathbb{N}$ such that: for every run $v \in L(A)$, letting $k_{v}$ be the number of faults in $v, C(\Pi(v)) \in\left\{k_{v}-1, k_{v}\right\}$. An automaton $A$ is fault countable if there exists a fault counter of $A$.

Given an automaton $A, q, q^{\prime} \in Q, k \in \mathbb{N}$ and $a \in \Sigma_{o}$, we write $q \rightarrow_{A}^{a, k} q^{\prime}$ if there is a path in $A$ from $q$ to $q^{\prime}$ of unobservable events except for the last transition labeled by $a$ with $k$ faulty transitions.

Proposition 6 Assuming that there is no vanishing repairs/faults in A, deciding if $A$ is fault countable w.r.t. $F$ is in NLOGSPACE.

Proof Let $A=\left(S, \Sigma, T, s_{0}\right)$ be an automaton. As there is no vanishing repairs/faults, if $q \rightarrow_{A}^{a, k} q^{\prime}$, then $k \leq 1$. We build the following variant of the twin automaton $A_{C}=$ $\left(S_{C}, \Sigma, T_{C},\left\{s_{0}, s_{0}, 0\right\}\right)$ where:

$$
\begin{aligned}
& \text { - } S_{C}=S \times S \times\{-1,0,1, \perp\}, \\
& \text { - }\left(\left(q_{1}, q_{2}, n\right), a,\left(q_{1}^{\prime}, q_{2}^{\prime}, m\right)\right) \in T_{C} \text { iff } q_{1} \rightarrow_{A}^{a, k} q_{1}^{\prime}, q_{2} \rightarrow_{A}^{a, k^{\prime}} q_{2}^{\prime} \text { and } \\
& \quad \text { - if } n=\perp \text {, then } m=\perp, \\
& \quad \text { - if } n \neq \perp \text { and } n+k-k^{\prime} \in\{-1,0,1\} \text {, then } m=n-k+k^{\prime}, \\
& \quad \text { - if } n \neq \perp \text { and } n+k-k^{\prime} \notin\{-1,0,1\} \text {, then } m=\perp .
\end{aligned}
$$

This construction is of size at most $4 .|A|^{2}$. Remark that the third component of the automaton keeps the difference of the number of faults by the two followed runs as long as this difference is not strictly greater than 1 . It is set to $\perp$ if the difference gets bigger than 1 .

We will now show that $A$ is count diagnosable if and only if no state of the form $\left(q, q^{\prime}, \perp\right)$ is reachable, which can be tested in NLOGSPACE.

Let us suppose that there exists $q, q^{\prime} \in S$ such that $\left(q, q^{\prime}, \perp\right)$ is reachable. Let $v$ be a run reaching $\left(q, q^{\prime}, \perp\right)$. We suppose $\left(q, q^{\prime}, \perp\right)$ to be the first state of $v$ which third component is a $\perp$. By construction of our twin product, we can associate $v$ to two runs $v_{1}$ and $v_{2}$ of $A$ with same observation and such that one of the two runs (say $v_{1}$ ) contains at least two more faults than the other. Consequently, suppose there exists a fault counter $C$, let $k$ be the number of faults in $v_{1}$ ( $v_{2}$ has thus less than $k-2$ faults), let $c=C\left(\Pi\left(v_{1}\right)\right)$, then $c \leq k-2$ and $c \geq k-1$ by definition of a fault counter, which is not possible. Therefore $A$ is not fault countable. 
Conversely, suppose $A$ is not fault countable. Then there exists $w \in \Sigma_{o}^{*}$ such that $w$ is the projection of several runs of $A$, for which no correct estimation of the number of faults (up to one missing fault occurrence) can be given from observation $w$. By definition of a fault counter, this means that there exists two runs $v_{1}$ and $v_{2}$ such that $w=\Pi\left(v_{1}\right)=\Pi\left(v_{2}\right)$ and the number of faults in $v_{1}$ is at least two more than the number of fault in $v_{2}$. Let $v$ be the run in $A_{C}$ following the two runs $v_{1}$ and $v_{2}$. This run ends in a state $\left(s^{+}\left(v_{1}\right), s^{+}\left(v_{2}\right), \perp\right)$. Thus a state of $A_{C}$ which third component is $\perp$ is reachable.

Remark that this proof does not immediately give the construction of a fault counter for the automaton. We will say that an automaton is T-Diagnosable w.r.t. $N$ if repairs can be faithfully detected. Intuitively, this property can be checked by inversion of safe and faulty states, an then checking T-diagnosability of the so-obtained system. Consider the Diagnosis function $\Delta: L_{o}(A) \rightarrow\{N, F, U\}$ defined by (2).

We define the function $\sharp_{\Delta}^{F}$ from $L_{o}(A)$ to $\mathbb{N}$ as follows: Let $\mu \in L_{o}(A)$ and $\rho \in$ $(N+U+F)^{*}$ the associated sequence of verdict emitted by $\Delta$. Let $\rho^{\prime} \in(N+F)^{*}$ be the projection of $\rho$ on the verdicts $\{N, F\}$, then $\sharp_{\Delta}^{F}(\mu)$ is the number of occurrences of pairs $N F$ that appear in $\rho^{\prime}$. Intuitively, $\sharp_{\Delta}^{F}$ is a function that will be used to count the number of faults the diagnoser is able to detect. We can define similarly function $\sharp_{\Delta}^{N}$, counting the number of detected repairs, by inverting $N$ and $F$ in the previous definition.

Given a run $u$ of $A, \sharp_{A}^{F}(u)$ denotes the number of times $A$ moves from a normal state to a faulty state in $u$ and $\sharp_{A}^{N}(\sigma(u))$ denotes the number of times $A$ evolves from a faulty state to a normal state in $u$. We can now state the following proposition:

Proposition 7 If $A$ is T-Diagnosable w.r.t. $F$ and T-Diagnosable w.r.t. $N$, and has no vanishing faults nor repairs, then $\forall v \in L(A)$ and $\mu=\Pi(v)$, then

$-0 \leq \sharp_{A}^{F}(v)-\sharp_{\Delta}^{F}(\mu) \leq 1$.

$-0 \leq \sharp_{A}^{N}(v)-\sharp_{\Delta}^{N}(\mu) \leq 1$.

Moreover if $\Delta(\mu)=F$ then $\sharp_{A}^{F}(v)=\sharp_{\Delta}(\mu)$ and if $\Delta(\mu)=N$ then $\sharp_{A}^{N}(v)=\sharp_{\Delta}^{N}(\mu)$.

Proof We proceed by induction. The base case is $v=\varepsilon$. In this case, $\sharp_{A}^{N}(v)=0$ and $\sharp_{\Delta}^{N}(\Pi(v))=0$ as $s_{o} \in N$ and $\Delta(\Pi(v)) \in\{N, U\}$ whereas $\sharp_{A}^{F}(v)=0$ and $\sharp_{\Delta}^{F}(\Pi(v))=0$.

Let us now consider a sequence

$$
v=v_{1}^{N} v_{1}^{F} \cdots v_{k}^{N} v_{k}^{F} \in L(A)
$$

such that $\forall i \leq k$,

- $v_{1}^{N} v_{1}^{F} \cdots v_{i-1}^{F} \longrightarrow v_{1}^{N} v_{1}^{F} \cdots v_{i-1}^{F} v_{i}^{N} \in L_{N}(A)$, and

$-v_{1}^{N} v_{1}^{F} \cdots v_{i}^{N} \longrightarrow v_{1}^{N} v_{1}^{F} \cdots v_{i-1}^{F} v_{i}^{N} v_{i}^{F} \in L_{F}(A)$

and assume that

$-0 \leq \sharp_{A}^{F}(v)-\sharp_{\Delta}^{F}(\Pi(v)) \leq 1$. 
$-0 \leq \sharp_{A}^{N}(v)-\sharp_{\Delta}^{N}(\Pi(v)) \leq 1$.

and that if $\Delta(\Pi(v))=F$ then $\sharp_{A}^{F}(s)=\sharp_{\Delta}^{F}(\Pi(v))$ and if $\Delta(\Pi(v))=N$ then $\sharp_{A}^{N}(s)=$ $\sharp_{\Delta}^{N}(\Pi(v))$.

Consider now $v^{\prime}=v v_{k+1}^{N} v_{k+1}^{F} \in L(A)$ and let $s_{k} \in S$ be the unique state reached by triggering $v$ in $A$. By definition $s_{k} \in F$ and the state reached by triggering the first event in $v_{k+1}^{N}$ belongs to $N$. As $A$ has no vanishing-repair, $\left|\Pi\left(v_{k+1}^{N}\right)\right| \geq 1$. Let $u_{k+1}^{N}$ be the the minimal prefix of $v_{k+1}^{N}$ that ends with an observable. We then have two different cases

- either $\Delta\left(\Pi\left(v u_{k+1}^{N}\right)\right)=N$ and we have

$$
\sharp_{A}^{N}\left(v u_{k+1}^{N}\right)=\sharp_{\Delta}^{N}\left(\Pi\left(v u_{k+1}^{N}\right)\right)=k+1
$$

as $\sharp_{A}^{N}(v)=\sharp_{\Delta}^{N}(\Pi(v))=k$, and as A is T-Diagnosable w.r.t F, $\Delta(\cdot)$ emitted the verdict $F$ after $\Pi(v)$ while observing a sub-sequence of $v_{k}^{F}$. Thus $\sharp_{\Delta}^{N}(\cdot)$ is incremented by 1 as well as $\sharp_{A}^{N}(\cdot)$ as the sequence leads to a repair state.

- or $\left.\left.\Delta\left(\Pi\left(v u_{k+1}^{N}\right)\right)\right)\right)=U$, meaning that the diagnoser is still not able to say that the system is repaired and in that case $\sharp_{A}^{N}\left(v u_{k+1}^{N}\right)=\sharp_{\Delta}^{N}\left(\Pi\left(v u_{k+1}^{N}\right)\right)+1$. However, as $A$ is T-Diagnosable w.r.t. $N$, there exists a prefix $u^{N} \in \Sigma^{*} \Sigma_{o}$ such that $u_{k+1}^{N} \leq$ $u^{N} \leq v_{k+1}^{N}$, and $\left.\left.\Delta\left(\Pi\left(v u^{N}\right)\right)\right)\right)=N$. At this point $\sharp_{A}^{N}\left(v u^{N}\right)=\sharp_{\Delta}^{N}\left(\Pi\left(v u^{N}\right)\right)=k+1$. For the remaining sub-sequence up to $v_{k+1}^{N}$ either the diagnoser can only emit the verdict $N$ or the verdict $U$ as the corresponding sequence is not faulty and thus the function $\sharp_{\Delta}^{N}()$ is not incremented.

The proof showing that the function $\sharp_{\Delta}^{F}()$ is also incremented by 1 after $v \cdot v_{k+1}^{N}$ while reading $v_{k+1}^{F}$ is symmetric to the previous case by replacing $N$ by $F$ and vice-versa.

Now, consider the case where $v=v_{1}^{N} v_{1}^{F} \cdots v_{k-1}^{F} \cdot v_{k}^{N} \in L(A)$ ends on a normal state. We have $\Delta(v) \in\{N, U\}$, and $s_{k} \in N$.

Consider the extension of $v$ with a sequence of faulty states $v_{k+1}^{F}$. As $s_{k}$ is in $N$, as $v_{k+1}^{F}$ starts with a faulty state and as we have no vanishing repair and A is T-diag w.r.t $N$, we have $\sharp_{A}^{N}(v)=\sharp_{\Delta}^{N}(v)$. As $v_{k+1}^{F}$ is a sequence of faulty states, $\sharp_{\Delta}^{N}(v)=\sharp_{\Delta}^{N}\left(v \cdot v_{k+1}^{F}\right)$ We hence have $\sharp_{A}^{N}\left(v \cdot v_{k+1}^{F}\right)=\sharp_{\Delta}^{N}(v)=k$, and the property is still satisfied.

Intuitively, this proposition states that we can build from the diagnoser a function that counts the number of times the system becomes faulty (resp. is repaired) with a difference of at most 1 . Furthermore, the difference is null as soon as the fault (resp repair) is diagnosed by the diagnoser. Note that the condition for counting in proposition 7 is sufficient, but not necessary as shown by the automaton of Figure 14 .

In this example, the automaton is T-diagnosable w.r.t. $\mathrm{N}$ but not w.r.t. F, moreover the sequence of verdicts emitted by $\Delta$ is $N U U N$. However, after reading $a a$ we know a single fault happened for sure. 


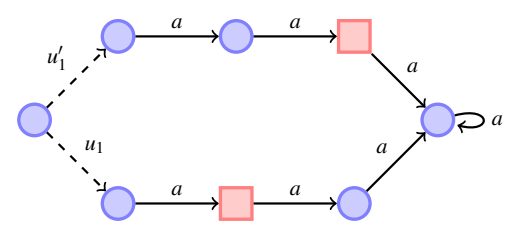

Fig. 14 A T-Diagnosable automaton wr.t. $\mathrm{N}$ but not w.r.t. $\mathrm{F}$

\section{Related work}

The diagnosis of such transient faults has been considered in [3], which proposed four notions of diagnosability. One of them ("O-diagnosability") consists in detecting the occurrence of a transient fault, even after it has been repaired, which amounts to saturating $L_{F}(A)$ (see Section 2.2). Symmetrically, the "I-diagnosability" aims at detecting the occurrence of a repair, even if fault(s) followed, which amounts to inverting the roles of $S_{F}$ and $S_{N}$, or to saturating the safe language $L_{N}(A)$. Both notions thus match the standard (or historical) notion of diagnosability for a slightly modified version of $A$.

In [7], two definitions involving multiple occurrences of faults are given. A system is $K$-diagnosable if the execution of any state-trace containing at least $\mathrm{K}$ failures can be deduced within a finite delay from the observed behavior. $K$-diagnosability is not monotonic, and the authors also introduce $[1 \cdots K]$-diagnosability, that is met by systems that are $J$-diagnosable for every $1 \leq J \leq K$. Compared with $[1 \cdots K]$ diagnosability or simply $K$ diagnosability, we introduced a sufficient condition under which it is possible to count exactly the number of faults that occurred in the system. Furthermore, similarly to [3], the definitions of diagnosability introduced in [7], do not request the detection of the fault before its repair.

In the same manner, [3] introduced the notions of "P-diagnosability" and "Rdiagnosability". These two notions are dual: P-diagnosability states that after the occurrence of a fault, it is always possible to detect the fact that the system is currently faulty, based on the observation (even though the fault has been repaired in the past). Conversely, $R$-diagnosability states that after a fault is repaired, it is possible to detect in finite time whether the system is currently in a safe state. As we are mainly detecting fault occurrences, our work should only be compared to the notion of "P-diagnosability". Our notion of T-Diagnosability is then stronger than Pdiagnosability, as we require that detection of faults occur before they are repaired. It is then easy to show that whenever a system is T-diagnosable then it is also Pdiagnosable. We now express the notion of "P-diagnosability" in our context (i.e. using a state-based approach (Note that a similar definition has been recently proposed in [1])) and show that deciding whether an automaton $A$ is P-diagnosable is a PSPACE complete problem. Formally, we have:

Definition 1 An automaton $A$ is $P$-diagnosable w.r.t. $\Sigma_{o}, F$ iff

$$
\begin{gathered}
\exists n \in \mathbb{N}, \forall v_{1} \in L_{F}^{\min }(A), \forall v_{1} v_{2} \in L(A), \\
{\left[\left|v_{2}\right|_{o} \geq n \Rightarrow \exists v_{2}^{\prime} \leq v_{2}: \Pi^{-1} \circ \Pi\left(v_{1} v_{2}^{\prime}\right) \subseteq L_{F}(A)\right]}
\end{gathered}
$$


The authors of [3] are interested in the dynamic behaviour of discrete event systems where failure and reset events occur continuously along any path of the system's evolution. To represent this, they introduce the notions of $\Sigma_{f}$-recurrence and $\Sigma_{r}$-recurrence.

An automaton $A$ is $\Sigma_{f}$-recurrent iff

$$
\begin{gathered}
\exists n \in \mathbb{N}, \forall v_{1} \in L_{F}^{\min }(A), \forall v_{1} v_{2} \in L(A), \\
{\left[\left|v_{2}\right|_{o} \geq n \Rightarrow \exists v_{2}^{\prime} \leq v_{2}: v_{1} v_{2}^{\prime} \subseteq L_{N}(A)\right]}
\end{gathered}
$$

We denote by $L_{R}^{\min }=\left\{v \alpha \in L_{N}(A) \mid v \in L_{F}(A) \wedge \alpha \in \Sigma\right\}$ the set of minimal repaired words of $A$, i.e. words that correspond to a run ending with a transition from a faulty state to a normal one in $A$. An automaton $A$ is $\Sigma_{r}$-recurrent iff

$$
\begin{gathered}
\exists n \in \mathbb{N}, \forall v_{1} \in L_{R}^{\min }(A), \forall v_{1} v_{2} \in L(A), \\
{\left[\left|v_{2}\right|_{o} \geq n \Rightarrow \exists v_{2}^{\prime} \leq v_{2}: v_{1} v_{2}^{\prime} \subseteq L_{F}(A)\right]}
\end{gathered}
$$

Under those restrictions, they obtained the following decidability result.

Proposition 8 ([3]) Given a $\Sigma_{f}$-recurrent and $\Sigma_{r}$-recurrent automaton A, and assuming there is no vanishing fault, P-diagnosability of A can be decided in PSPACE.

Note that, in [1], the authors propose another algorithm to decide the P-diagnosability of a system $A$ based on the classical notion of twin-plant. However the tests that are necessary to check the P-diagnosability requires to check all the faulty runs that are equivalents in this machine, leading to a PSPACE algorithm for the test.

We complete these results by proving the PSPACE-hardness of $P$-Diagnosability. The proof reuses ingredient from the proof of hardness of T-diagnosability (Thm. 2) with some subtle differences.

Proposition 9 Given a $\Sigma_{f}$-recurrent and $\Sigma_{r}$-recurrent automaton A with no vanishing fault, P-diagnosability of A is PSPACE-hard.

Proof The proof shows hardness of the problem by reduction from a language inclusion problem, which is known to be PSPACE-complete. The language inclusion problem can be formulated as follows: given $A_{0}, \ldots, A_{n}$ some deterministic finite automata, does $\bigcap_{i \in 0 . . n} L\left(A_{i}\right)=\emptyset$ ?

Let $n \in \mathbb{N}$ and for $0 \leq i \leq n, A_{i}=\left(S_{i}, \Gamma, T_{i}, q_{i n}^{i}, F_{i}\right)$ be some deterministic finite automaton on alphabet $\Gamma$. We build the finite automaton $A=\left(S, \Sigma, T, q_{0}\right)$ (see Figure 15) where:

- $\Sigma=\Gamma \cup\left\{u_{0}, \ldots u_{n}\right\} \cup\{f, \sharp, b\}$

- $S=\left\{q_{0}\right\} \cup\left\{q_{i}^{j} \mid i, j \in \in^{0 . . n}\right\} \cup\left\{q_{i n}^{j} \mid j \in 0 . . n\right\} \cup \cup_{0 \leq i \leq n} S_{i}$

- $T=\left\{\left(q_{0}, u_{j}, q_{i n}^{j}\right) \mid j \in 0 . . n\right\} \cup\left\{\left(q_{n}^{j}, \sharp, q_{0}^{j} \mid j \in 0 . . n\right\} \cup\left\{\left(q, b, q_{0}^{j}\right) \mid q \in F_{j}, j \in 0 . . n\right\} \cup\right.$

$\left\{\left(q_{i}^{j}, \sharp, q_{i+1}^{j} \mid i=0 . . n, j=0 \ldots n-1\right\} \cup \bigcup_{0 \leq j \leq n} T_{j}\right.$

The set of safe states is $S_{F}=\left\{q_{i}^{i} \mid i=0 \ldots n\right\}$. The set of faulty states is $S \backslash S_{F}$. We set $\Sigma_{o}=\Gamma \cup\{\sharp\}$.

We claim that $A$ is P-diagnosable if and only if $\bigcap_{i \in 0 . . n} L\left(A_{i}\right)=\emptyset$. 


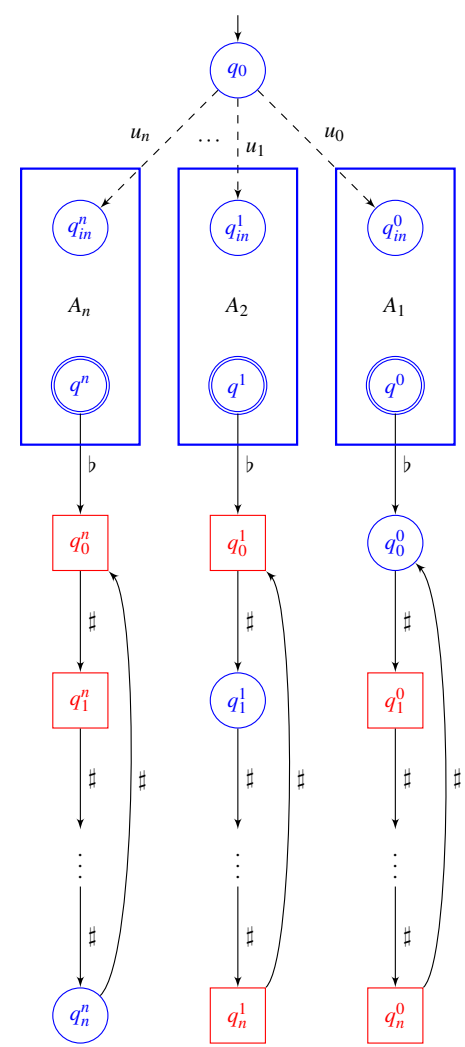

Fig. 15 PSPACE-hardness of P-diagnosability. Red states are faulty, and blue states normal states.

First, remark that after observing $w b \sharp^{m}$ for $m \in \mathbb{N}$, the current run leads the automaton in some state $q_{k}^{j}$ with $j \leq n, k \leq n$ and $k=m \bmod (n+1)$ such that $A_{j}$ accepts $w$.

Suppose that $A$ is P-diagnosable. Let $w \in L\left(A_{0}\right), v_{1}$ be the unique run of $A$ such that $\sigma\left(v_{1}\right)=u_{0} w b \sharp$. This run is unique as $u_{0}$ leads to the initial state of the deterministic automaton $A_{0}$. As $v_{1}$ is a minimal faulty run, there exists $m \in \mathbb{N}$ such that the run $v_{1} v_{2}$ with $\sigma\left(v_{1} v_{2}\right)=u_{0} w b \sharp^{m}$ verifies $\Pi^{-1} \circ \Pi\left(v_{1} v_{2}\right) \subseteq L_{F}(A)$. From our initial remark, as $q_{k}^{k}$ with $k \leq n$ and $k=m \bmod (n+1)$ is safe, it means that $A_{k}$ does not accept $w$. As this is true for every $w \in L\left(A_{0}\right), \bigcap_{i \in 0 . . n} L\left(A_{i}\right)=\emptyset$.

Conversely suppose that $\bigcap_{i \in 0 . . n} L\left(A_{i}\right)=\emptyset$. Let $v_{1}$ be a minimal faulty run. $v_{1}$ is of the form $\sigma\left(v_{1}\right)=u_{i} w b \sharp^{m}$ with $i \leq n$ and $w \in L\left(A_{i}\right)$. As $\bigcap_{i \in 0 . . n} L\left(A_{i}\right)=\emptyset$, there exists $j \leq n$ such that $w \notin L\left(A_{j}\right)$. Let $m_{j} \in \mathbb{N}$ be such that $j=m+m_{j} \bmod n$ and consider the run $v_{1} v_{2}$ with $\sigma\left(v_{1} v_{2}\right)=u_{i} w b \sharp^{m+m_{j}}$. Thanks to our initial remark, as $q_{j}^{r}$ with $r \neq j$ is a faulty state and $A_{j}$ does not accept $w, \Pi^{-1} \circ \Pi\left(v_{1} v_{2}\right) \in L_{F}(A)$. Thus the fault can be claimed. As this is true for every minimal faulty run $v_{1}, A$ is $\mathrm{P}$-diagnosable. 


\section{Conclusion}

We have proposed a notion of "timely-diagnosability" that requires the detection (in bounded time) of transient faults after they occur, and before they are repaired. T-diagnosability for a deterministic partially observed automaton is decidable (in PSPACE). T-diagnosability is stronger than the P-diagnosability of [3] in the sense that the latter does not require that a transient fault be detected before it is repaired. Nevertheless, P-Diagnosability remains PSPACE complete, as shown in this paper. For deterministic systems that do not contain vanishing faults nor repairs, checking T-Diagnosability amounts to detecting ambiguous cycles and occurrences of repairs before any possible diagnosis. The question of whether a system contains vanishing faults (resp. repairs) is decidable with reasonable complexity. Requiring a system to be free from vanishing faults and repairs is quite a sensible restriction, and when a system does not satisfy this property, it can be corrected to avoid considering vanishing faults as real faults. Now, while determinism allows one to express diagnosability properties in terms of faulty and safe languages, it leads to quite complicated criteria for T-diagnosability, as in Theorem 1.

As future line of research, it could be interesting to define T-diagnosability for non-deterministic automata, and to explore whether criteria simplify. For example, it is likely that in the absence of vanishing faults and of vanishing repairs, T-diagnosability is preserved by $\Sigma_{o}$-closure. Also, while the T-diagnosability of faults relies on a complicated criterion, it is likely that systems which are both T-diagnosable for faults and for repairs are much easily characterized. This subclass is quite interesting, as it corresponds to systems where all changes of the status of the system from safe to faulty and conversely are detected in bounded time, and in any case before they change again. So ambiguity, when it appears, can not last forever.

Besides these immediate perspectives, the future of this work is definitely in the direction of quantitative analysis. Being able to characterize exactly, after a bounded delay, in which state class lies system $A$ is a very strong property. A more relevant question would be to determine how likely it is that $A$ is in $S_{N}$ or $S_{F}$ given partial observations, and whether this relative certainty passes some threshold in a bounded time after that status of a system $A$ has changed.

\section{Acknowledgements}

The authors would like to thank Francois Godi, Xavier Montillet and Chen Qian, master students at ENS Rennes, for interesting discussions that led to this work.

\section{References}

1. Ab. Boussif, B. Liu, and M. Ghazel. A twin-plant based approach for diagnosability analysis of intermittent failures. In 13th International Workshop on Discrete Event Systems, pages 237-244, Xi'an, China, 2016.

2. F. Cassez and S. Tripakis. Fault diagnosis with static and dynamic diagnosers. Fundamenta Informaticae, 88(4):497-540, November 2008. 
3. Olivier Contant, Stéphane Lafortune, and Demosthenis Teneketzis. Diagnosis of intermittent faults. Discrete Event Dynamic Systems, 14(2):171-202, 2004.

4. E. Fabre, L. Hílouet, E. Lefaucheux, and H. Marchand. Diagnosability of repairable faults. In 13th International Workshop on Discrete Event Systems, pages 256-262, Xi'an, China, 2016.

5. T. Jéron, H. Marchand, S. Pinchinat, and M-O. Cordier. Supervision patterns in discrete event systems diagnosis. In Workshop on Discrete Event Systems, WODES'06, pages 262-268, Ann-Arbor (MI, USA), July 2006.

6. S. Jiang, Z. Huang, V. Chandra, and R. Kumar. A polynomial time algorithm for diagnosability of discrete event systems. IEEE Transactions on Automatic Control, 46(8):1318-1321, 2001.

7. S. Jiang, R. Kumar, and H.E. Garcia. Diagnosis of repeated/intermittent failures in discrete event systems. IEEE Transactions on Robotics and Automation, 19(2):310-323, April 2003.

8. Dexter Kozen. Lower bounds for natural proof systems. In 18th Annual Symposium on Foundations of Computer Science, Providence, Rhode Island, USA, 31 October - 1 November 1977, pages 254-266. IEEE Computer Society, 1977.

9. Klaus-Jörn Lange and Peter Rossmanith. The emptiness problem for intersections of regular languages. In Mathematical Foundations of Computer Science 1992, 17th International Symposium, MFCS'92, Prague, Czechoslovakia, August 24-28, 1992, Proceedings, pages 346-354, 1992.

10. Meera Sampath, Raja Sengupta, Stéphane Lafortune, Kasim Sinnamohideen, and Demosthenis Teneketzis. Failure diagnosis using discrete-event models. IEEE Trans. Contr. Sys. Techn., 4(2):105124, 1996.

11. Walter J. Savitch. Relationships between nondeterministic and deterministic tape complexities. $J$. Comput. Syst. Sci., 4(2):177-192, 1970.

12. James C. Tiernan. An efficient search algorithm to find the elementary circuits of a graph. Commun. ACM, 13(12):722-726, 1970.

13. J. Zaytoon and S. Lafortune. Overview of fault diagnosis methods for discrete event systems. Annual Reviews in Control, 37(2):308-320, 2013. 